\title{
Neutron multiplication in random media: reactivity and kinetics parameters
}

\author{
Coline Larmier ${ }^{\mathrm{a}}$, Andrea Zoia ${ }^{\mathrm{a}, *}$, Fausto Malvagi ${ }^{\mathrm{a}}$, Eric Dumonteil ${ }^{\mathrm{b}}$, Alain \\ Mazzolo $^{\mathrm{a}}$ \\ ${ }^{a} D E N$-Service d'études des réacteurs et de mathématiques appliquées (SERMA), CEA, \\ Université Paris-Saclay, F-91191, Gif-sur-Yvette, France \\ ${ }^{b}$ IRSN, 31 Avenue de la Division Leclerc, 92260 Fontenay aux Roses, France.
}

\begin{abstract}
Eigenvalue problems for neutron transport in random geometries are key for many applications, ranging from reactor design to criticality safety. In this work we examine the behaviour of the reactivity and of the kinetics parameters (the effective delayed neutron fraction and the effective neutron generation time) for three-dimensional UOX and MOX assembly configurations where a portion of the fuel pins has been randomly fragmented by using various mixing statistics. For this purpose, we have selected stochastic tessellations of the Poisson, Voronoi and Box type, which provide convenient models for the random partitioning of space, and we have generated an ensemble of assembly realizations; for each geometry realization, criticality calculations have been performed by using the Monte Carlo code TRIPOLI- $4{ }^{\circledR}$, developed at CEA. We have then examined the evolution of the ensemble-averaged observables of interest as a function of the average chord length of the random geometries, which is roughly proportional to the correlation length of the fuel fragmentation. The methodology proposed in this work is fairly general and could be applied, e.g., to the assessment of re-criticality probability following severe accidents.
\end{abstract}

Keywords: Random media, Monte Carlo, TRIPOLI- ${ }^{\circledR}$, Kinetics parameters, Markov tessellations, Poisson, Voronoi.

\footnotetext{
*Corresponding author

Email address: andrea. zoia@cea.fr (Andrea Zoia)
} 


\section{Introduction}

Neutron multiplication in stochastic media has attracted intense research efforts, in view of many relevant applications emerging in reactor physics and criticality safety, such as the design of prismatic and pebble-bed reactors with double

5 heterogeneity fuel (Murata et al., 1996; Liang et al., 2013; Brown and Martin, 2004), the analysis of neutron absorbers with dispersed poison grains (Doub, 1961) or MOX fuels with Pu-rich agglomerates (Yamamoto, 2010), optimal radioactive waste storage (Williams, 2003), and the assessment of the safety margins due to the multiplication factor distribution (Pomraning, 1999; Williams, 2000; Williams and Larsen, 2001), only to name a few.

The formal approach to these problems consists in averaging the critical Boltzmann equation with respect to the random configurations, and then solving the corresponding eigenvalue problem (Pomraning, 1991, 1999). Theoretical progress on such topics is hindered by the great amount of ingenuity required to 15 derive exact results, even in the simplest models and configurations (Pomraning, 1999; Williams, 2000, 2003; Williams and Larsen, 2001). Perturbation theory can be helpful, but several simplifications are usually required, including monoenergetic transport, isotropic scattering, or diffusion approximation (Pomraning, 1999; Williams and Larsen, 2001).

20 In this respect, Monte Carlo simulation offers a convenient tool for the numerical analysis of eigenvalue problems in stochastic media. For this purpose, two complementary strategies have been proposed (Pomraning, 1991): the former consists in generating by Monte Carlo methods a collection of realizations of random media (obeying some given distribution, which is supposed to accurately model the system under analysis) and then solving the eigenvalue problem for each realization by using a transport code. The ensemble averages will finally yield the moments, and in principle also the full distribution, of the physical observables of interest, e.g., the multiplication factor or the neutron flux shape. Such reference solutions are exact, since the stochastic nature of the medium is 30 entirely preserved, including the effects on neutron trajectories possibly induced by the spatial correlations. The latter consists in developing effective transport kernels capable of reproducing on-the-fly the 'average' behaviour of neutron displacement accounting for the underlying random heterogeneities, such as in the case of the well-known Chord Length Sampling algorithm (Zimmerman,

35 1990; Zimmerman and Adams, 1991; Donovan and Danon, 2003; Donovan et al., 2003). In this case, a single eigenvalue calculation with effective jump distribution is sufficient, at the expense of sacrificing the correlations (whose effects must be typically neglected in constructing these algorithms). 
Reference solutions, although computationally expensive, are nonetheless of utmost importance for the validation of approximate, albeit much faster methods, and for the verification of exact formulas (Levermore et al., 1986; Adams et al., 1989; Malvagi et al., 1992; Su and Pomraning, 1995; Zuchuat et al., 1994; Larsen and Vasques, 2011; Brantley, 2011; Donovan and Danon, 2003; Donovan et al., 2003; Brantley and Palmer, 2009; Brantley, 2009). Significant advances have been made in the numerical simulation of eigenvalue problems in the presence of stochastic inclusions, where objects (typically spheres) are randomly placed within a background matrix (Murata et al., 1996; Liang et al., 2013; Brown and Martin, 2004). Stochastic inclusions emerge for instance in the modelling of the double heterogeneities in prismatic or pebble-bed reactors. In particular, highly sophisticated algorithms have been devised in order to properly take into account boundary effects due to spheres not entirely contained in the medium (Grieshiemer et al., 2010; Ji and Martin., 2013). Eigenvalue calculations in stochastic tessellations, where the medium is supposed to be partitioned into a collection of random (fissile and non-fissile) volumes obeying a given mixing statistics (Pomraning, 1991), have been limited so far to one-dimensional configurations of the rod or slab type (Pomraning, 1999; Williams and Larsen, 2001). Such models might represent, e.g., the accidental positioning of fuel lumps into moderating material, in the context of criticality safety.

In a series of recent papers, we have examined the statistical properties of linear particle transport through $d$-dimensional stochastic tessellations without multiplication (Lepage et al., 2011; Larmier et al., 2017a). In particular, we have computed reference solutions for the ensemble-averaged scalar flux and the reflection and transmission probabilities for Poisson (Markov) mixing statistics by revisiting the benchmark originally proposed by Adam, Larsen and Pomraning (Adams et al., 1989; Brantley, 2011; Brantley and Palmer, 2009; Brantley, 2009), and we have then extended our findings to the case of Voronoi and Box statistics (Larmier et al., 2017b).

In this work, we will adopt stochastic tessellations (Santalo, 1976; Torquato, 2013) in order to assess the impact of the three-dimensional random fragmentation of fuel elements on the key safety parameters for criticality calculations, including the multiplication factor $k_{\text {eff }}$, the effective delayed neutron fraction $\beta_{\text {eff }}$, and the effective neutron generation time $\ell_{\text {eff }}$. In-pile fuel degradation might result from partial core melt-down during severe accidents, with melting, resolidification and relocation (Hagen and Hofmann, 1987; Hofmann, 1999), as 75 occurred in the case of the Three Mile Island unit 2 (Broughton et al., 1989). The effects of such scenario on neutron kinetics are of utmost importance for the 
evaluation of the re-criticality probability. To our best knowledge, the influence of random geometries on kinetics parameters has never been addressed before. Starting from a reference UOX or MOX assembly with $17 \times 17$ fuel pins, we will consider three perturbed configurations having the central pin, $7 \times 7$ central pins and the whole $17 \times 17$ pins being randomly fragmented. We will assume that the random re-arrangement after melt-down can be described by ternary mixing statistics (Pomraning, 1991), accounting for the dispersion of the fuel, the cladding and the moderator, the average linear size of the chunks for each material being a free parameter of the model. For each realization, we will perform criticality calculations by using the Monte Carlo transport code TRIPOLI- $4{ }^{\circledR}$ developed at CEA (Brun et al., 2015), so as to investigate the distribution of $k_{\text {eff }}$, $\beta_{e f f}$ and $\ell_{e f f}$ as a function of the model parameters, including the material compositions, the kind of stochastic tessellation, the linear size of the random chunks, and the number of fragmented fuel pins.

In order to better grasp the physical behaviour of these systems without being hindered by the complexity of all the ingredients involved in nuclear accidents, the analysis of assembly configurations that will be carried out in this work is admittedly highly simplified with respect to the realistic description of fuel degradation: for instance, we will focus exclusively on neutron transport, and we will not include the effects due to thermal-hydraulics, thermo-mechanics or the complex physical-chemical reactions occurring in accidental transients (Hagen and Hofmann, 1987; Hofmann, 1999). Nonetheless, the methodology proposed in this paper is fairly broad and can be applied without any particular restrictions to more sophisticated models.

This paper is organized as follows: in Sec. 2 we will briefly introduce the stochastic tessellations that will be used in this work. Then, in Sec. 3 we will describe the benchmark model for the partially melted fuel assembly. Simulation results for the multiplication factor and the kinetics parameters will be presented and discussed in Sec. 4. Conclusions will be finally drawn in Sec. 5.

\section{Stochastic tessellations}

Stochastic tessellations form a convenient class of probabilistic models to partition a given $d$-dimensional domain into random polyhedral cells (Santalo, 1976; Chiu et al., 2013; Torquato, 2013), and as such have been adopted in a broad spectrum of applications, ranging from material science (Gilbert, 1962; Meijering, 1953) to stereology (Serra, 1982; Santalo, 1976): for an extensive review, see, e.g., (Santalo, 1976; Miles, 1972; Torquato, 2013). In this section, we introduce the three mixing statistics that will be used in order to generate the 
random geometries for the criticality calculations. In particular, we will recall the algorithms for their construction based on Monte Carlo methods, and their main statistical features. These algorithms have already been discussed elsewhere, but they are reported here in order for the paper to be self-contained. For the sake of conciseness, the three stochastic tessellations will be distinguished by their label $m$, namely, $m=\mathscr{P}$ for Poisson tessellations, $m=\mathscr{V}$ for Voronoi tessellations, and $m=\mathscr{B}$ for Box tessellations. The algorithms described in the following have been implemented into a computer code that can perform the statistical analysis of an ensemble of realizations, and can produce input files for the TRIPOLI-4 ${ }^{\circledR}$ Monte Carlo transport code (Brun et al., 2015).

\subsection{Isotropic Poisson tessellations}

Markovian mixing is generated by using isotropic Poisson geometries, which form a prototype process of stochastic tessellations: a domain included in a $d$-dimensional space is partitioned by randomly generated $(d-1)$-dimensional hyper-planes drawn from an underlying Poisson process (Santalo, 1976; Miles, 1970, 1972). An algorithm has been recently proposed for finite $d$-dimensional geometries (Serra, 1982; Ambos and Mikhailov, 2011), allowing for the explicit construction of three-dimensional homogeneous and isotropic Poisson tessellations. In the following we will present the algorithm for the construction of these geometries restricted to a cubic box (further details are provided in (Larmier et al., 2016)).

The algorithm starts by sampling a random number of hyper-planes $N_{H}$ from a Poisson distribution of intensity $4 \rho_{\mathscr{P}} R$, where $R$ is the radius of the sphere circumscribed to the cube and $\rho_{\mathscr{P}}$ is the (arbitrary) density of the tessellation, carrying the units of an inverse length. This normalization of the density $\rho_{\mathscr{P}}$ corresponds to the convention used in (Santalo, 1976), and is such that $\rho_{\mathscr{P}}$ yields the mean number of $(d-1)$-hyperplanes intersected by an arbitrary segment of unit length. Then, we generate the planes that will cut the cube. We choose a radius $r$ uniformly in the interval $[0, R]$ and then sample two additional parameters, namely, $\xi_{1}$ and $\xi_{2}$, from two independent uniform distributions in the interval $[0,1]$. A unit vector $\mathbf{n}=\left(n_{1}, n_{2}, n_{3}\right)^{T}$ with components

$$
\begin{aligned}
& n_{1}=1-2 \xi_{1} \\
& n_{2}=\sqrt{1-n_{1}^{2}} \cos \left(2 \pi \xi_{2}\right) \\
& n_{3}=\sqrt{1-n_{1}^{2}} \sin \left(2 \pi \xi_{2}\right)
\end{aligned}
$$

is generated. Denoting by $\mathbf{M}$ the point such that $\mathbf{O M}=r \mathbf{n}$, the random plane will finally obey $n_{1} x+n_{2} y+n_{3} z=r$, passing trough $\mathbf{M}$ and having normal vector $\mathbf{n}$. 
By construction, this plane does intersect the circumscribed sphere of radius $R$ but not necessarily the cube. The procedure is iterated until $N_{H}$ random planes have been generated. The polyhedra defined by the intersection of such random planes are convex.

\subsection{Poisson-Voronoi tessellations}

Voronoi tessellations refer to another prototype process for isotropic random division of space (Santalo, 1976). A portion of a space is decomposed into polyhedral cells by a partitioning process based on a set of random points, called 'seeds'. From this set of seeds, the Voronoi decomposition is obtained by applying the following deterministic procedure: each seed is associated with a Voronoi cell, defined as the set of points which are nearer to this seed than to any other seed. Such a cell is convex, because obtained from the intersection of half-spaces.

In this paper, we will exclusively focus on Poisson-Voronoi tessellations, which form a subclass of Voronoi geometries (Meijering, 1953; Gilbert, 1962; Miles, 1972). The specificity of Poisson-Voronoi tessellations concerns the sampling of the seeds. In order to construct Poisson-Voronoi tessellations restricted to a cubic box of side $L$, we adopt the algorithm proposed in (Miles, 1972). First, we choose the random number of seeds $N_{S}$ from a Poisson distribution of parameter $\left(\rho_{\mathscr{V}} L\right)^{3}$, where $\rho_{\mathscr{V}}$ characterizes the density of the tessellation. Then, $N_{S}$ seeds are uniformly sampled in the box $[-L / 2, L / 2]^{3}$. For each seed, we compute the corresponding Voronoi cell as the intersection of half-spaces bounded by the mid-planes between the selected seed and any other seed. In order to avoid confusion with the Poisson tessellations described above, we will mostly refer to Poisson-Voronoi geometries simply as Voronoi tessellations in the following.

\subsection{Poisson Box tessellations}

Box tessellations form a class of anisotropic stochastic geometries, composed of rectangular boxes with random sides. For the special case of Poisson Box tessellations (as proposed by (Miles, 1972)), a domain is partitioned by $i$ ) randomly generated planes orthogonal to the $x$-axis, through a Poisson process of intensity $\rho_{x}$; ii) randomly generated planes orthogonal to the $y$-axis, through a Poisson process of intensity $\rho_{y}$; $\left.i i i\right)$ randomly generated planes orthogonal to the $z$-axis, through a Poisson process of intensity $\rho_{z}$. In the following, we will assume that the three parameters are equal, namely, $\rho_{x}=\rho_{y}=\rho_{z}=\rho_{\mathscr{B}}$.

In order to tessellate a cube of side $L$, the construction algorithm is the following: we begin by sampling a random number $N_{x}$ from a Poisson distribution of intensity $\rho_{\mathscr{B}} L$. Then, we sample $N_{x}$ points uniformly on the segment 
$[-L / 2, L / 2]$. For each point of this set, we cut the geometry with the plane orthogonal to the $x$-axis and passing through this point. We repeat this process for the $y$-axis and the $z$-axis. For the sake of conciseness, we will denote by Box tessellations these Poisson Box tessellations. Poisson-Box tessellations have the remarkable property that their chord length distribution is almost identical to that of the homogeneous and isotropic Poisson tessellations (Ambos and Mikhailov, 2011; Larmier et al., 2017b). They could then be used as an approximate model for Markov mixing statistics, in view of the extremely reduced algorithmic complexity required for their construction.

\subsection{Statistical properties of infinite tessellations}

The observables of interest associated to the stochastic geometries, such as for instance the volume of a polyhedron, its surface, the number of faces, and so on, are random variables. With a few remarkable exceptions, their exact distributions are unfortunately unknown (Santalo, 1976). Nevertheless, exact results have been established for some low-order moments of the observables, in the limit case of domains having an infinite extension (Santalo, 1976; Chiu et al., 2013; Miles, 1970).

In this respect, Poisson tessellations have been shown to possess a remarkable property: in the limit of infinite domains, an arbitrary line will be cut by the hyperplanes of the tessellation into chords whose lengths are exponentially distributed with parameter $\rho_{\mathscr{P}}$ (whence the identification with Markovian mixing) (Santalo, 1976; Miles, 1970, 1972). Thus, in this case, the average chord length $\langle\Lambda\rangle_{\infty}$ satisfies $\langle\Lambda\rangle_{\infty}=\rho_{\mathscr{P}}^{-1}$, and its probability density $\Pi^{\mathscr{P}}(\Lambda)$ is given by

$$
\Pi^{\mathscr{P}}(\Lambda)=\frac{1}{\langle\Lambda\rangle_{\infty}} e^{-\Lambda /\langle\Lambda\rangle_{\infty}}
$$

To the best of our knowledge, the exact distribution of the chord length for Voronoi and Box tessellations is not known. However, the average chord length for Voronoi and Box tessellations has been rigorously derived (Santalo, 1976; Miles, 1970, 1972) and is recalled in Tab. 1. Monte Carlo simulations show that the chord length distribution for the Box tessellations is actually very close (and nonetheless not equal) to that of Poisson tessellations (Larmier et al., 2017b). The shape of the chord length distribution for Voronoi tessellations is on the contrary very different from the exponential shape.

\subsection{Chord lengths and finite-size effects}

200 In the presence of boundaries, the average chord lengths of finite-size stochastic tessellations will generally differ from their asymptotic values. In order to investigate such effects, we have numerically computed by Monte Carlo simulation 


\begin{tabular}{cc}
\hline$m$ & $\langle\Lambda\rangle_{\infty}$ \\
\hline $\mathscr{P}$ & $\rho_{\mathscr{P}}{ }^{-1}$ \\
$\mathscr{V}$ & $0.6872 \rho_{\mathscr{V}}{ }^{-1}$ \\
$\mathscr{B}$ & $(2 / 3) \rho_{\mathscr{B}}{ }^{-1}$ \\
\hline
\end{tabular}

Table 1: Exact formulas for the average chord length $\langle\Lambda\rangle_{\infty}$ in infinite tessellations, for different mixing statistics $m$. Expressions are taken from (Santalo, 1976; Miles, 1972).

the distribution and the average of the chord length for each mixing statistics $m$. A random tessellation is first generated, and a line is then drawn with an isotropic and homogeneous distribution (technically speaking, the line must obey the $\mu$ randomness (Coleman, 1969)). The intersections of the line with the polyhedra of the geometry are computed, and the resulting segment lengths are recorded. This step is repeated for a large number of random lines. Then, a new geometry is generated and the whole procedure is iterated for several geometries, in order to get satisfactory statistics.

The numerical results for the normalized average chord length $\left\langle\Lambda^{*} \mid L^{*}\right\rangle=$ $\left\langle\Lambda \mid L^{*}\right\rangle /\langle\Lambda\rangle_{\infty}$ as a function of the normalized linear size $L^{*}=L /\langle\Lambda\rangle_{\infty}$ of the domain are illustrated in Fig. 1 for different mixing statistics $m$. Monte Carlo simulation results for the chord length distribution are shown in Fig. 2, for $\langle\Lambda\rangle_{\infty}=1$ and for several values of $L$. For small $L$, finite-size effects are visible in the chord length distribution: indeed, the longest length that can be drawn across a box of linear size $L$ is $\sqrt{3} L$, which thus induces a cut-off on the distribution. For large $L$, the finite-size effects due to the cut-off fade away. In particular, the probability density for Poisson tessellations eventually converges to the expected exponential behaviour. Simulations show that the chord length distributions in Box tessellations and in Poisson tessellations are very close, which is consistent with the observations in (Ambos and Mikhailov, 2011). On the contrary, in Voronoi tessellations, the probability density has a distinct non-exponential functional form (see Fig. 2).

\subsection{Assigning material compositions: colored geometries}

In order to describe the fuel pin fragmentation that will be discussed in the next section, the material compositions of the fuel pin components must be (randomly) transferred to the stochastic tessellation. For the sake of simplicity, we will assume that only three compositions are present, namely the fuel, the 


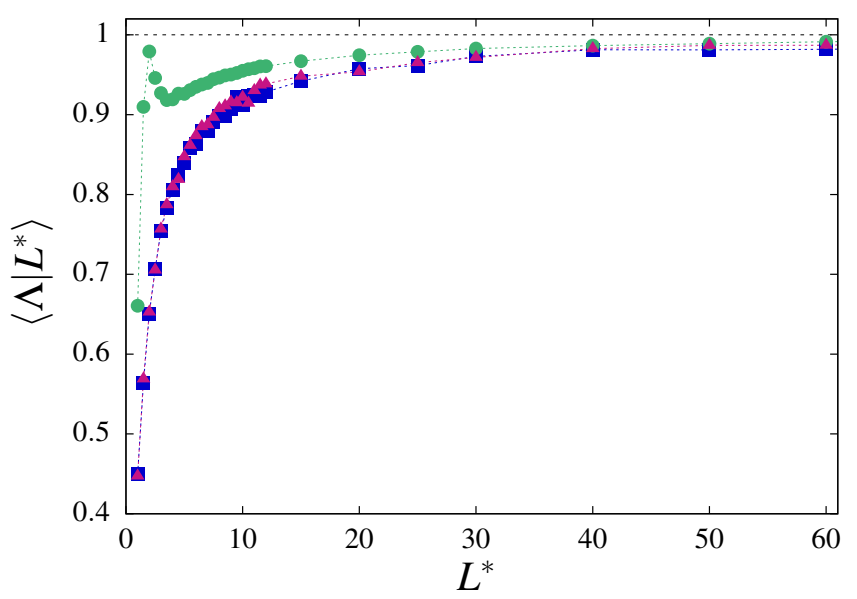

Figure 1: Average chord length $\left\langle\Lambda \mid L^{*}\right\rangle$ (arbitrary units) as a function of the rescaled system size $L^{*}=L /\langle\Lambda\rangle_{\infty}$, with parameter $\langle\Lambda\rangle_{\infty}=1$. Blue squares denote the results for Poisson tessellations, red triangles for Box tessellations, and green circles for Voronoi tessellations. Dotted lines have been added to guide the eye. Dashed black line corresponds to the asymptotic value for infinite tessellations.

230 cladding and the moderator. This procedure can be achieved by adopting ternary stochastic mixtures, which are realized as follows. First, Poisson, Voronoi or Box tessellations are constructed as described above. Then, each polyhedron of the geometry is assigned a material composition by formally attributing a distinct 'label' (also called 'color'), say ' $\alpha$ ', ' $\beta$ ' or ' $\gamma$ ', with associated probabilities $p_{\alpha}$, ${ }_{235} p_{\beta}$ and $p_{\gamma}=1-p_{\alpha}-p_{\beta}$. We will call a 'cluster' the collection of adjacent polyhedra sharing the same label.

After assigning colors to stochastic geometries, we can introduce the average chord length through clusters with label $i(i=\alpha, \beta$ or $\gamma)$, denoted by $\left\langle\Lambda_{i}\right\rangle_{\infty}$. For infinite tessellations, it can be shown that $\left\langle\Lambda_{i}\right\rangle_{\infty}$ is related to the average chord length $\langle\Lambda\rangle_{\infty}$ of the geometry via

$$
\langle\Lambda\rangle_{\infty}=\left(1-p_{i}\right)\left\langle\Lambda_{i}\right\rangle_{\infty}
$$

This property stems from the binomial distribution of the coloring procedure (Haran et al., 2000; Larmier et al., 2016), and holds true for each tessellation $m$. Additionally, the corresponding probability density $\Pi^{\mathscr{P}}\left(\Lambda_{i}\right)$ is still exponential for 


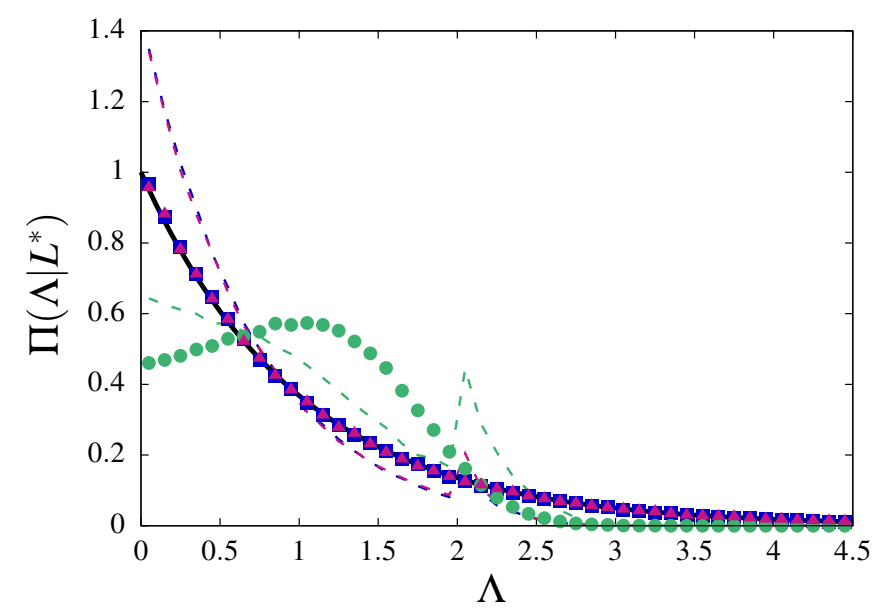

Figure 2: The probability density $\Pi(\Lambda)$ of the chord length for $\langle\Lambda\rangle_{\infty}=1$ as a function of the system size $L$ ( $\Lambda$ and $L$ are given in arbitrary units). Results for Poisson tessellations are displayed in blue, Box tessellations in red, and Voronoi tessellations in green. Dashed lines correspond to $L=2$. Symbols correspond to $L=100$. The exponential distribution is shown as a black solid line, for reference.

infinite Poisson geometries, i.e.,

$$
\Pi^{\mathscr{P}}\left(\Lambda_{i}\right)=\frac{1}{\left\langle\Lambda_{i}\right\rangle_{\infty}} e^{-\Lambda_{i} /\left\langle\Lambda_{i}\right\rangle_{\infty}}
$$

For Voronoi and Box tessellations, the full probability densities $\Pi^{\mathscr{V}}\left(\Lambda_{i}\right)$ and $\Pi^{\mathscr{B}}\left(\Lambda_{i}\right)$ are not known.

\section{A model of assembly with fragmented fuel pins}

The stochastic tessellations described above can be conveniently adopted to represent a partially melted fuel assembly, the size of the fuel fragments being determined by the geometry density (which is a free parameter of the model). We propose in the following some benchmark configurations that are simple enough to enable a physical interpretation of the effects induced by the presence of random material fragmentation, and yet retain the key ingredients.

As a reference configuration we will consider an assembly composed of $17 \times$ 17 square fuel pin-cells of side length $\delta=1.262082 \mathrm{~cm}$ in the plane $O_{x y}$ and 


\begin{tabular}{|c|c|c|}
\hline Material & Isotopes & $\begin{array}{c}\text { Concentration } \\
\left(\text { atoms } \times 10^{24} \times \mathrm{cm}^{-3}\right)\end{array}$ \\
\hline \multicolumn{3}{|l|}{ UOX fuel } \\
\hline & U235 & $8.4148 \times 10^{-4}$ \\
\hline & $\mathrm{U} 238$ & $2.1625 \times 10^{-2}$ \\
\hline & O16 & $4.4932 \times 10^{-2}$ \\
\hline \multicolumn{3}{|l|}{ MOX fuel } \\
\hline & $\mathrm{U} 234$ & $3.9390 \times 10^{-7}$ \\
\hline & $\mathrm{U} 235$ & $4.9524 \times 10^{-5}$ \\
\hline & U238 & $2.1683 \times 10^{-2}$ \\
\hline & PU238 & $2.2243 \times 10^{-5}$ \\
\hline & PU239 & $7.0164 \times 10^{-4}$ \\
\hline & PU240 & $2.7138 \times 10^{-4}$ \\
\hline & PU241 & $1.3285 \times 10^{-4}$ \\
\hline & PU242 & $6.6984 \times 10^{-5}$ \\
\hline & AM241 & $1.2978 \times 10^{-5}$ \\
\hline & AM242M & $2.2569 \times 10^{-10}$ \\
\hline & O16 & $4.5882 \times 10^{-2}$ \\
\hline \multicolumn{3}{|l|}{ Moderator } \\
\hline & $\mathrm{H} 1$ & $4.7716 \times 10^{-2}$ \\
\hline & O16 & $2.3858 \times 10^{-2}$ \\
\hline & B10 & $3.9724 \times 10^{-6}$ \\
\hline & B11 & $1.5890 \times 10^{-5}$ \\
\hline \multicolumn{3}{|l|}{ Cladding } \\
\hline & ZR90 & $2.2060 \times 10^{-2}$ \\
\hline & ZR91 & $4.8107 \times 10^{-3}$ \\
\hline & ZR92 & $7.3532 \times 10^{-3}$ \\
\hline & ZR94 & $7.4518 \times 10^{-3}$ \\
\hline & ZR96 & $1.2005 \times 10^{-3}$ \\
\hline
\end{tabular}

Table 2: Material compositions for the UOX and MOX assemblies used for the benchmark configurations.

of height $L_{z}=10 \mathrm{~cm}$. Reflective boundary conditions will be imposed on all sides of the assembly. The fuel elements will be entirely either of the UOX or MOX type: the respective material compositions for the fuel, the cladding and the moderator are provided in Tab. 2. The proposed compositions correspond to fresh (Beginning Of Life) fuel. The assembly will be assumed to be at a uniform temperature of $T=300 \mathrm{~K}$, for conservatism (Doppler effect on reactivity will be reduced).

The partial melting of a collection of fuel pins is then introduced by applying a stochastic ternary mixing model of Poisson, Voronoi or Box type to a central region composed of $n_{x} \times n_{y}$ cells. For the sake of simplicity, this region will be assumed to be located at the center of the assembly, with $n_{x}=n_{y}=n, n$ being odd. The assembly portion corresponding to this region is removed and replaced 
by a stochastic tessellation. The tessellation is then randomly 'colored' with ternary labels, namely, ' $\mathscr{F}$ ' for fuel, ' $\mathscr{C}$ ' for cladding and ' $\mathscr{M}$ ' for moderator, with corresponding coloring probabilities $p_{\mathscr{F}}, p_{\mathscr{C}}$ and $p_{\mathscr{M}}$ chosen so that for each material $i$ the ensemble-averaged volumic ratio $\left\langle p_{i}\right\rangle$ coincides with that of a pin-cell before fragmentation:

$$
\begin{gathered}
p_{\mathscr{F}}=\pi R_{1}^{2} / \delta^{2} \approx 0.335861 \\
p_{\mathscr{C}}=\pi\left(R_{2}^{2}-R_{1}^{2}\right) / \delta^{2} \approx 0.107943 \\
p_{\mathscr{M}}=\left(\delta^{2}-\pi R_{2}^{2}\right) / \delta^{2} \approx 0.556196 .
\end{gathered}
$$

Moreover, in order for the three stochastic tessellation models to yield comparable results with respect to neutron transport, we have set the free parameters of each model (i.e., the geometry density $\rho$ ) so as to have exactly the same average chord length $\langle\Lambda\rangle_{\infty}$. In-pile and out-of-pile experiments of core degradation show that the fuel fragments after melting are partially mixed with the cladding (Hagen and Hofmann, 1987; Hofmann, 1999): nonetheless, for the present benchmark we assume that the fuel and the cladding are present in distinct phases. The pin-cells surrounding the perturbed region are left unchanged.

It is important to note that, for a single geometrical realization, the volumic ratio of material $i$ in the tessellation is not rigorously equal to $p_{i}$, because of finite-size effects. However, the finite-size effects progressively fade away with increasing fragmentation of the tessellation, and become negligible for tessellations dense enough. This behaviour will be discussed in Sec. 4.8.

For our benchmark model, we have selected three fragmented configurations, each corresponding to a different size for the melted portion of the assembly: in configuration 1 , only the central pin-cell is replaced by a ternary mixing $(n=1)$. In configuration 2 , we have chosen a portion $n=7$, i.e., about half of the assembly is fragmented. Finally, in configuration 3, the entire assembly is fragmented $(n=17)$. For illustration, some of the resulting partially melted assemblies are shown in Figs. 3 and 4.

275 The physical observables that we would like to determine are the ensembleaveraged multiplication factor $\left\langle k_{\text {eff }}\right\rangle$, the ensemble-averaged kinetics parameters (namely, the effective neutron generation time $\left\langle\ell_{\text {eff }}\right\rangle$ and the effective delayed neutron fraction $\left\langle\beta_{\text {eff }}\right\rangle$ ), as well as and the ensemble-averaged scalar particle flux $\langle\varphi(\mathbf{r}, E)\rangle$ within the assembly.

Our goal is to investigate how these physical observables are affected by the presence of the fragmented fuel pins. For this purpose, we will vary the mixing statistics by separately testing Poisson, Voronoi and Box tessellations, and the average chord length $\langle\Lambda\rangle_{\infty}$ for each tessellation (which basically rules the aver- 


\section{Configuration 1}
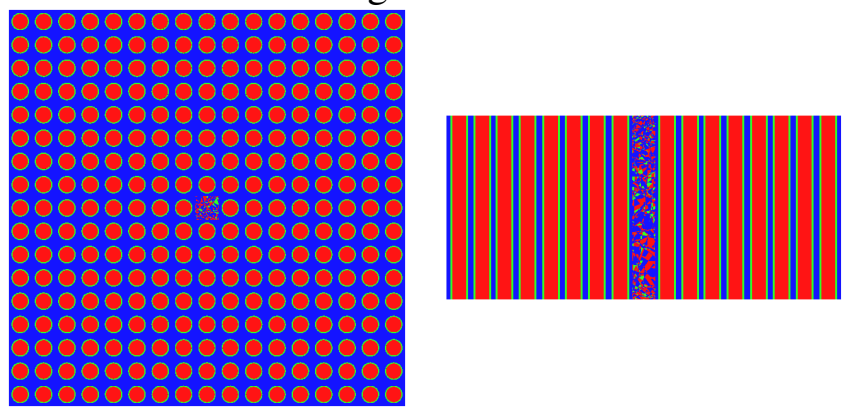

Configuration 2
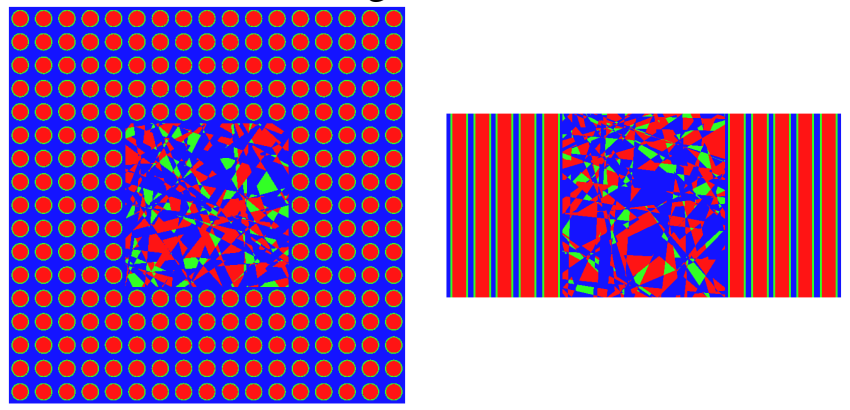

Configuration 3
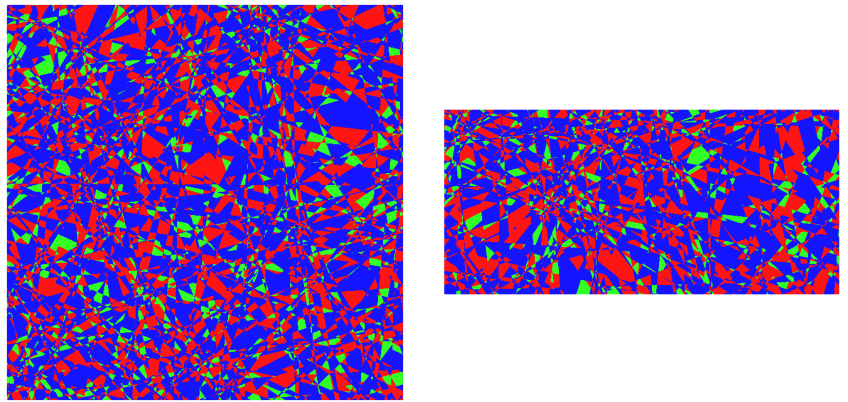

Figure 3: Assemblies with Poisson tessellation for the central fuel pins. Top: configuration $1(n=1)$, with $\langle\Lambda\rangle_{\infty}=0.05 \mathrm{~cm}$. Center: configuration $2(n=7)$, with $\langle\Lambda\rangle_{\infty}=0.2 \mathrm{~cm}$. Bottom: configuration $3(n=17)$, with $\langle\Lambda\rangle_{\infty}=0.15 \mathrm{~cm}$. Left column: radial view. Right column: axial view.

age size of the material chunks composing the randomized portion of the assembly). In-core experiments have shown that the fragment size may vary between less than a millimetre and several millimetres, depending on the re-solidification 
Poisson

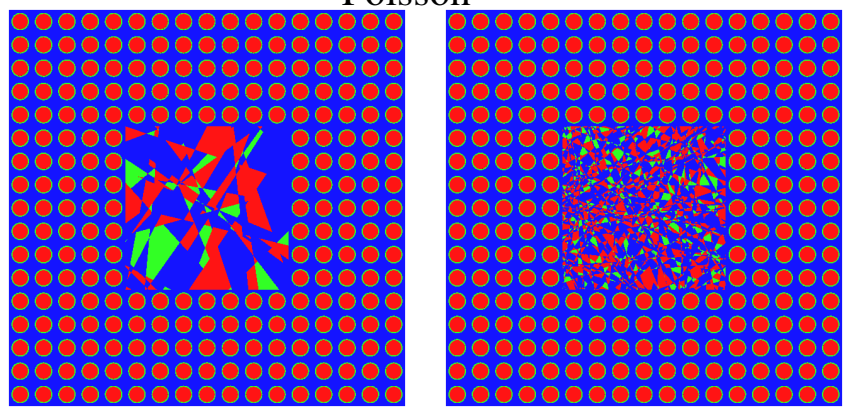

Voronoi
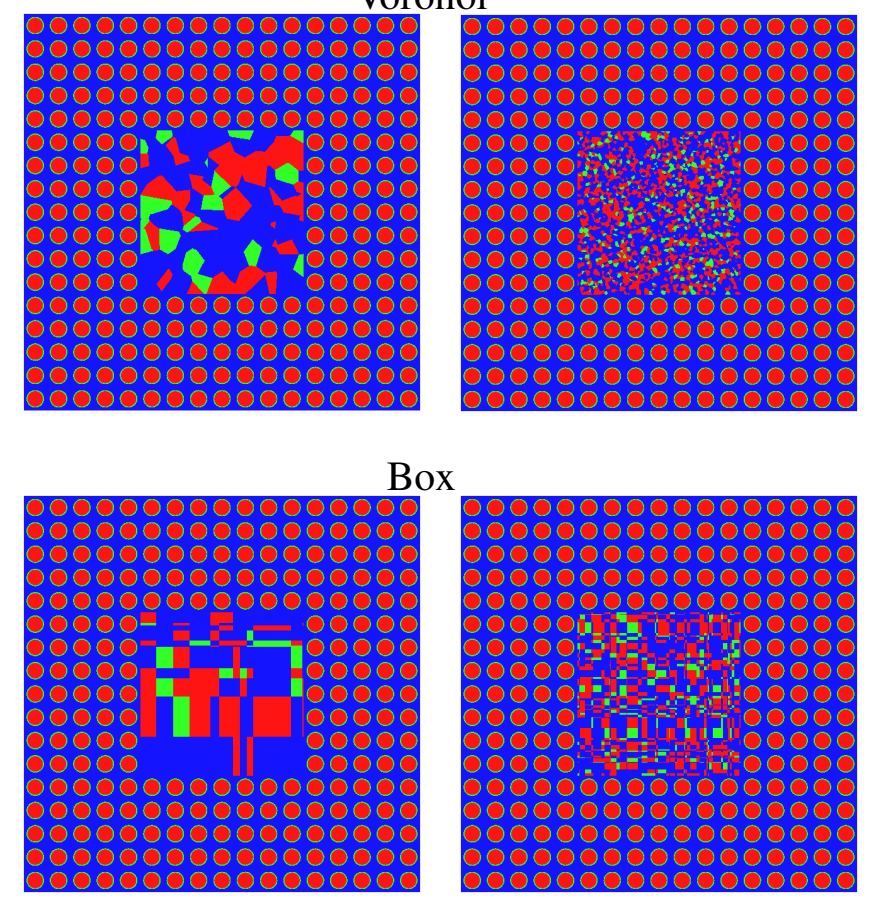

Figure 4: Assemblies with stochastic tessellation for the central fuel pins. Radial view of the configuration $2(n=7)$, for different mixing statistics and average chord lengths $\langle\Lambda\rangle_{\infty}$. Top: Poisson tessellation. Center: Voronoi tessellation. Bottom: Box tessellation. Left column: $\langle\Lambda\rangle_{\infty}=0.5 \mathrm{~cm}$. Right column: $\langle\Lambda\rangle_{\infty}=$ $0.1 \mathrm{~cm}$.

speed (Hagen and Hofmann, 1987; Broughton et al., 1989; Hofmann, 1999). Decreasing $\langle\Lambda\rangle_{\infty}$ means increasing the density of the tessellations, which implies 


\section{Simulation results}

In this section we present and discuss the criticality calculations performed with TRIPOLI- $4{ }^{\circledR}$ for the partially melted assemblies. The reference solutions for the ensemble-averaged multiplication factor $\left\langle k_{\text {eff }}\right\rangle$, kinetics parameters $\left\langle\ell_{\text {eff }}\right\rangle$ and $\left\langle\beta_{e f f}\right\rangle$, and scalar neutron flux $\langle\varphi(\mathbf{r}, E)\rangle$ have been computed as follows. For each assembly configuration, a large number $M$ of geometries has been generated, and the material properties have been attributed to each volume as described above. Then, for each realization $j$ of the ensemble, eigenvalue calculations have been carried out by using TRIPOLI- $4{ }^{\circledR}$. The number of simulated particle histories per configuration has been chosen so that the statistical error on the computed eigenvalue $k_{\text {eff }}$ is smaller than $50 \mathrm{pcm}$. For a given physical observable $\mathscr{O}$, the benchmark solution is obtained as the ensemble average

$$
\langle\mathscr{O}\rangle=\frac{1}{M} \sum_{j=1}^{M} \mathscr{O}_{j},
$$


where $\mathscr{O}_{j}$ is the Monte Carlo estimate for the observable $\mathscr{O}$ obtained for the $j$ th realization. The scalar flux $\varphi_{j}(\mathbf{r}, E)$ has been recorded by using the standard track length estimator over a pre-defined spatial grid.

The error affecting the average observable $\langle\mathscr{O}\rangle$ results from two separate contributions, namely, the dispersion

$$
\sigma_{G}^{2}=\frac{1}{M} \sum_{j=1}^{M} \mathscr{O}_{j}^{2}-\langle\mathscr{O}\rangle^{2}
$$

of the observables exclusively due to the stochastic nature of the geometries and of the material compositions, and

$$
\sigma_{\mathscr{O}}^{2}=\frac{1}{M} \sum_{j=1}^{M} \sigma_{\mathscr{O}_{j}}^{2},
$$

which is an estimate of the variance due to the stochastic nature of the Monte Carlo method for the particle transport, $\sigma_{\mathscr{O}_{j}}^{2}$ being the dispersion of a single calculation (Donovan and Danon, 2003; Donovan et al., 2003). The statistical error on $\langle\mathscr{O}\rangle$ is then estimated as

$$
\sigma[\langle\mathscr{O}\rangle]=\sqrt{\frac{\sigma_{G}^{2}}{M}+\sigma_{\mathscr{O}}^{2}} .
$$

Depending on the correlation lengths and on the volumetric fractions, the physical observables might display a larger or smaller dispersion around their average values. In order to assess the impact of such dispersion, we have also computed the full distribution of $k_{\text {eff }}$ based on the available realizations. The number of realizations $M$ has been adapted to the configuration (i.e., to the number $n$ of fragmented fuel pins) and to the chosen average chord length $\langle\Lambda\rangle_{\infty}$. As a general remark, decreasing the average chord length $\langle\Lambda\rangle_{\infty}$ for a given tessellation implies an increasing computational burden (each realization takes longer both for generation and for Monte Carlo transport), but also a better statistical averaging (a single realization is more representative of the 'typical' random behaviour). The parameter $M$ varies between $M=100$ for, e.g., $n=1$ and $\langle\Lambda\rangle_{\infty}=0.03 \mathrm{~cm}$, and $M=3000$ for, e.g., $n=17$ and $\langle\Lambda\rangle_{\infty}=3 \mathrm{~cm}$.

\subsection{The TRIPOLI-4 code and the simulation options}

TRIPOLI- $4{ }^{\circledR}$ is a general-purpose Monte Carlo radiation transport code developed at CEA and devoted to shielding, reactor physics with depletion, criticality safety and nuclear instrumentation (Brun et al., 2015). TRIPOLI- $4{ }^{\circledR}$ is 


$$
\text { Ka }
$$

\section{Kam actor} implementation has been chosen for IFP, with an arbitrary number of latent generations $M$ (Truchet et al., 2015). For all the simulations discussed here, we have chosen $M=20$.

360 For our simulations, we have largely benefited from a feature that has been recently implemented in the code TRIPOLI- $4^{\circledR}$, namely the possibility of exploiting pre-computed connectivity maps for the volumes composing the geometry. During the generation of the stochastic tessellations, care has been taken so as to store the indexes of the neighbouring volumes for each realization, which means that during the geometrical tracking a particle will have to find the following crossed volume in a list that might be considerably smaller than the total number of random volumes composing the box (depending on the features of the actor safety during transient operation and accidental excursions (Nauchi and number of random volumes composing the box (depending on the features of the

the reference Monte Carlo code for CEA (including laboratories and reactor facilities) and the French utility company EDF. It is also the reference code of the CRISTAL Criticality Safety package (Gomit et al., 2011) developed with IRSN and AREVA. The code offers both fixed-source and criticality simulation modes. Neutrons are simulated in the energy range from $20 \mathrm{MeV}$ to $10^{-5} \mathrm{eV}$. Particle transport is performed in continuous-energy, and the necessary nuclear data (i.e., point-wise cross-sections, scattering kernels, secondary energy-angle distributions, secondary particle yields, fission spectra, and so on) are read by the code from any evaluation written in ENDF-6 format (McLane, 2004). The code can irectly access files in ENDF and PENDF format.

For the criticality calculations presented in the following, we have selected the JEFF-3.1.1 nuclear data library (Santamarina et al., 2009). Concerning probability tables for the unresolved resonance range, TRIPOLI- $4{ }^{\circledR}$ adopts the CALENDF code (Sublet et al., 2011). Thermal data $S(\alpha, \beta)$ for bound hydrogen in water were available in JEFF-3.1.1 at $296 \mathrm{~K}$. Doppler broadening of elastic scattering differential cross sections has been enforced by using the standard SVT model. The DBRC model for resonant nuclides, although available in TRIPOLI-4 ${ }^{\circledR}$ (Zoia et al., 2012), has not been used, since it is not expected to ave a major impact on reactivity and kinetics parameters at low temperature.

Concerning kinetics parameters calculations, starting from version 4.10 the Iterated Fission Probability (IFP) method has been implemented in TRIPOLI$4{ }^{\circledR}$ (Truchet et al., 2015) and extensively validated (Truchet et al., 2015; Zoia and Brun, 2016; Zoia et al., 2016). Exact calculation of adjoint-weighted quantities by the IFP method establishes Monte Carlo simulation as a reference tool actor safety during transient opertion and accidental excurions (Nauchi and 


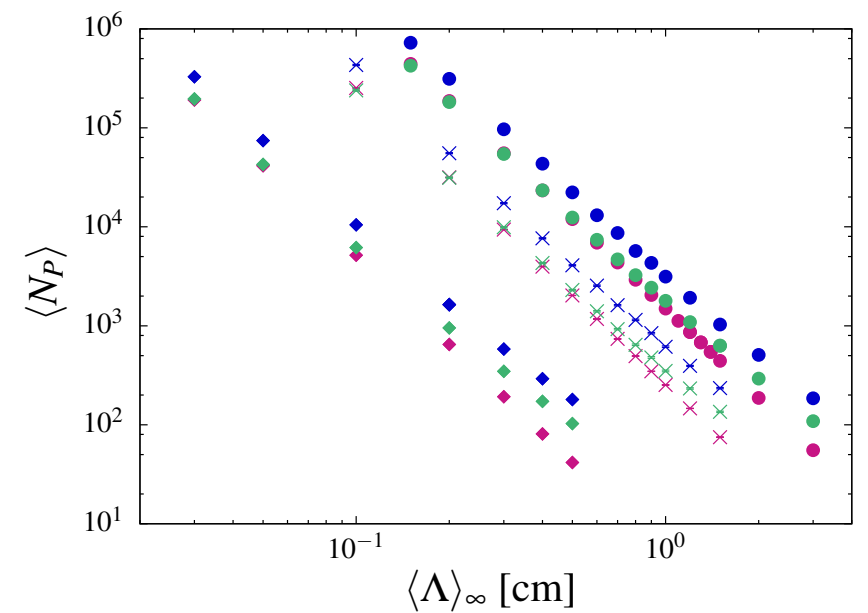

Figure 5: Average number of polyhedra $\left\langle N_{P}\right\rangle$ of the stochastic tessellation as a function of the average chord length $\langle\Lambda\rangle_{\infty}$ and of the mixing statistics $m$. Blue symbols denote the results for Poisson tessellations, red symbols for Box tessellations, and green symbols for Voronoi tessellations. Diamonds correspond to configuration $1(n=1)$, crosses to configuration $2(n=7)$ and circles to configuration $3(n=17)$.

random geometry).

\subsection{Complexity and computer time}

Before addressing the simulation results for the ensemble-averaged physical observables, we briefly analyse the computational cost of the performed calculations as a function of the complexity of the underlying stochastic tessellations. Transport calculations have been run on a cluster based at CEA, with Xeon E5$2680 \mathrm{~V} 22.8 \mathrm{GHz}$ processors. The average number of polyhedra $\left\langle N_{P}\right\rangle$ pertaining to each random geometry is reported in Fig. 5: it is apparent that the quantity $\left\langle N_{P}\right\rangle$ increases with decreasing $\langle\Lambda\rangle_{\infty}$, i.e., with increasing fragmentation. The scaling law is fairly independent of the mixing statistics $m$, and roughly goes as $\left\langle N_{P}\right\rangle \sim 1 /\langle\Lambda\rangle_{\infty}^{3}$ for any $m$. The exponent of the scaling law stems from the dimension $d=3$. The number $n$ of fragmented fuel pins does not affect these results, as expected.

The corresponding (ensemble-averaged) computer times for each assembly configuration are reported in Tab. 3. Dispersions $\sigma[t]$ are also given. The simulation time increases when increasing the portion of the assembly that is subject 


\begin{tabular}{ccccc}
\hline & & & $\min \langle\Lambda\rangle_{\infty}$ & $\max \langle\Lambda\rangle_{\infty}$ \\
\hline$n$ & $t_{\text {mix }}$ & $m$ & $\langle t\rangle \pm \sigma[t]$ & $\langle t\rangle \pm \sigma[t]$ \\
\hline \multirow{3}{*}{1} & \multirow{3}{*}{3260} & $\mathscr{P}$ & $6170 \pm 570$ & $2970 \pm 40$ \\
& & $\mathscr{V}$ & $4580 \pm 110$ & $2940 \pm 25$ \\
& & $\mathscr{B}$ & $4060 \pm 225$ & $2960 \pm 30$ \\
\hline \multirow{3}{*}{7} & \multirow{2}{*}{2990} & $\mathscr{P}$ & $18000 \pm 3000$ & $2840 \pm 60$ \\
& & $\mathscr{V}$ & $9200 \pm 150$ & $2820 \pm 30$ \\
& & $\mathscr{B}$ & $7500 \pm 1000$ & $2840 \pm 40$ \\
\hline \multirow{3}{*}{17} & \multirow{3}{*}{1350} & $\mathscr{P}$ & $48000 \pm 16000$ & $2200 \pm 200$ \\
& & $\mathscr{V}$ & $14000 \pm 300$ & $2300 \pm 200$ \\
& & $\mathscr{B}$ & $16000 \pm 3000$ & $2200 \pm 200$ \\
\hline
\end{tabular}

Table 3: Average computer time $\langle t\rangle$ (expressed in seconds) and the corresponding standard deviation $\sigma[t]$ for transport simulations in benchmark configurations $n=1, n=7$ and $n=17$ with UOX fuel, as a function of the mixing statistics $m$, for the minimal (respectively, maximal) value of the chord length $\min \langle\Lambda\rangle_{\infty}$ (respectively, $\max \langle\Lambda\rangle_{\infty}$ ). The computer time $t_{m i x}$ (expressed in seconds) for transport simulations corresponding to atomic mix fuel fragmentation is also displayed. For reference, the computer time for a transport simulation in the UOX assembly with intact fuel pins is equal to 3240 seconds.

to fragmentation, as expected. While a decreasing trend for $\langle t\rangle$ as a function of $385\langle\Lambda\rangle_{\infty}$ is clearly apparent, subtle effects due to correlation lengths and volume fractions for the material compositions come also into play, and strongly influence the average computer time. For some configurations, the dispersion $\sigma[t]$ may become very large, and even be comparable to the average $\langle t\rangle$. The chosen tessellation model visibly affects the computer time. Atomic mix simulations are based on a single homogenized realization, and the dispersion is thus trivially zero.

\subsection{Multiplication factor}

We begin our analysis by considering the behaviour of the multiplication factor $\left\langle k_{\text {eff }}\right\rangle$, whose evolution is illustrated in Figs. 6, 7, and 8 for UOX and MOX assemblies with $n=1, n=7$ and $n=17$ melted fuel pins, respectively. The computed value $\left\langle k_{\text {eff }}\right\rangle$ is displayed as a function of increasing chord length $\langle\Lambda\rangle_{\infty}$, for Poisson, Voronoi and Box tessellations. As detailed above, the error bar on $\left\langle k_{\text {eff }}\right\rangle$ results from the contribution of the Monte Carlo statistical error (which is of the order of about $50 \mathrm{pcm}$ for all simulations) and the dispersion 

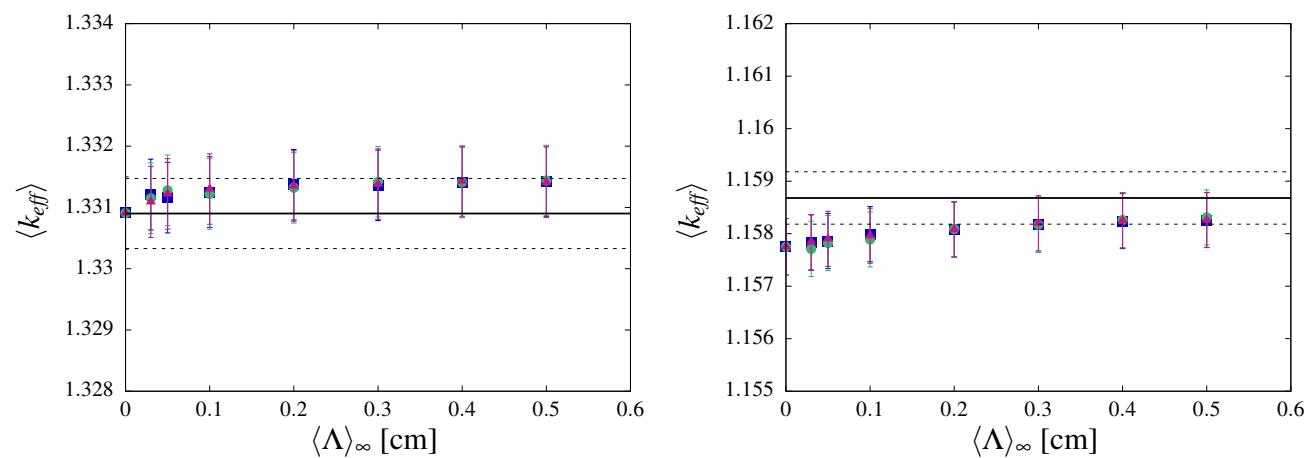

Figure 6: Evolution of the ensemble-averaged multiplication factor $\left\langle k_{\text {eff }}\right\rangle$ as a function of the average chord length $\langle\Lambda\rangle_{\infty}$. Left: UOX assembly with $n=1$. Right: MOX assembly with $n=1$. Blue squares denote the results for Poisson tessellations, red triangles for Box tessellations, and green circles for Voronoi tessellations. The limit case at $\langle\Lambda\rangle_{\infty}=0$ corresponds to the atomic mix model. The black solid line denotes $k_{\text {eff, },}$, the result for the assembly with intact fuel pins, which has been added for reference (dashed lines represent the $1 \sigma$ statistical uncertainty).

due to the random realizations. The limit case of atomic mix $\left(\langle\Lambda\rangle_{\infty} \rightarrow 0\right)$ is also shown. In each figure, the $k_{\text {eff, },}$ eigenvalue corresponding to an assembly with intact fuel pins is plotted for reference.

As expected on physical grounds, the impact of the stochastic tessellations on the multiplication factor depends on the size of the assembly that has been randomly fragmented. When $n=1$, the difference between $\left\langle k_{\text {eff }}\right\rangle$ and the reference $k_{e f f, 0}$ is of the order of $100 \mathrm{pcm}$, and falls almost within $1 \sigma$ uncertainty. The major contribution to the dispersion of the multiplication factor stems from the statistical error. In this case, the impact of the specific tessellations models is not appreciable. For UOX assemblies, the average values $\left\langle k_{\text {eff }}\right\rangle$ lie all slightly above $k_{\text {eff, },}$ for any $\langle\Lambda\rangle_{\infty}$, and seem to attain $k_{\text {eff, },}$ in the atomic mix limit. For MOX assemblies, the average values $\left\langle k_{\text {eff }}\right\rangle$ lie all slightly below $k_{\text {eff, },}$ for any $\langle\Lambda\rangle_{\infty}$, even in the atomic mix limit.

When $n=7$, a relevant portion of the fuel pins is fragmented, and the impact of the stochastic tessellations on the eigenvalue becomes apparent. For both UOX and MOX assemblies, in the atomic mix limit $\left\langle k_{\text {eff }}\right\rangle$ is well below the reference $k_{\text {eff, },}$ (about $1000 \mathrm{pcm}$ for UOX and $2000 \mathrm{pcm}$ for MOX). Then, for increasing chord length $\langle\Lambda\rangle_{\infty}$, for all the stochastic tessellations models $\left\langle k_{\text {eff }}\right\rangle$ 

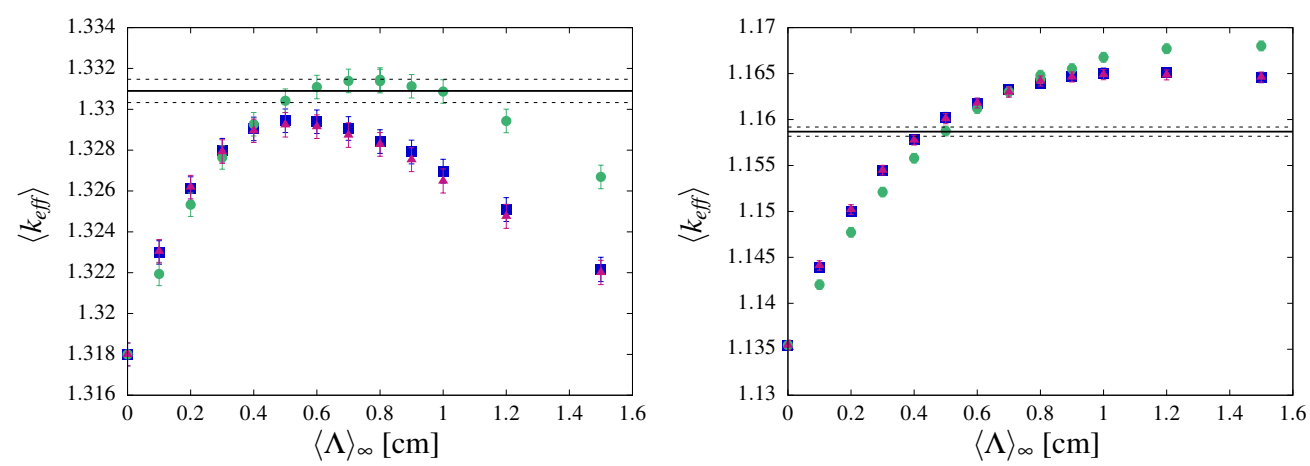

Figure 7: Evolution of the ensemble-averaged multiplication factor $\left\langle k_{\text {eff }}\right\rangle$ as a function of the average chord length $\langle\Lambda\rangle_{\infty}$. Left: UOX assembly with $n=7$. Right: MOX assembly with $n=7$. Blue squares denote the results for Poisson tessellations, red triangles for Box tessellations, and green circles for Voronoi tessellations. The limit case at $\langle\Lambda\rangle_{\infty}=0$ corresponds to the atomic mix model. The black solid line denotes $k_{\text {eff, },}$, the result for the assembly with intact fuel pins, which has been added for reference (dashed lines represent the $1 \sigma$ statistical uncertainty).

first increases up to a maximum value, and then decreases for even larger chord lengths. In the limit of very large $\langle\Lambda\rangle_{\infty}$, the stochastic tessellations would be trivially filled with a single material (fuel, cladding, or moderator), each appearing with its respective coloring probability. In this case, $\left\langle k_{e f f}\right\rangle$ would be the weighted sum of the multiplication factors of three configurations with the central portion of the assembly replaced by a fuel, cladding or moderator zone. The values $\left\langle k_{e f f}\right\rangle$ computed for Poisson and Box tessellations are almost indistinguishable, which supports our previous analysis. On the contrary, the $\left\langle k_{e f f}\right\rangle$ obtained for the Voronoi tessellations reach their maximum for a $\langle\Lambda\rangle_{\infty}$ larger than in the case of the other two tessellations. The $\left\langle k_{e f f}\right\rangle$ for Voronoi tessellations lie first below those of the Poisson and Box tessellations; after that $\left\langle k_{\text {eff }}\right\rangle$ has attained its maximum for the Poisson and Box tessellations, the values corresponding to Voronoi tessellations lie above the others. For the UOX assemblies, the maximum $\left\langle k_{e f f}\right\rangle$ for Poisson and Box tessellations (for $\langle\Lambda\rangle_{\infty} \sim 0.5 \mathrm{~cm}$ ) is about 300 pcm lower than $k_{\text {eff, } 0}$, whereas for Voronoi tessellations (for $\langle\Lambda\rangle_{\infty} \sim 0.7 \mathrm{~cm}$ ) is slightly higher. For MOX assemblies, the maxima are attained for larger average chord lengths $\left(\langle\Lambda\rangle_{\infty} \sim 1 \mathrm{~cm}\right.$ for Poisson and Box tessellations and $\Lambda \sim 1.6 \mathrm{~cm}$ for Voronoi tessellations) and are largely higher than the reference $k_{\text {eff }, 0}$ by about 

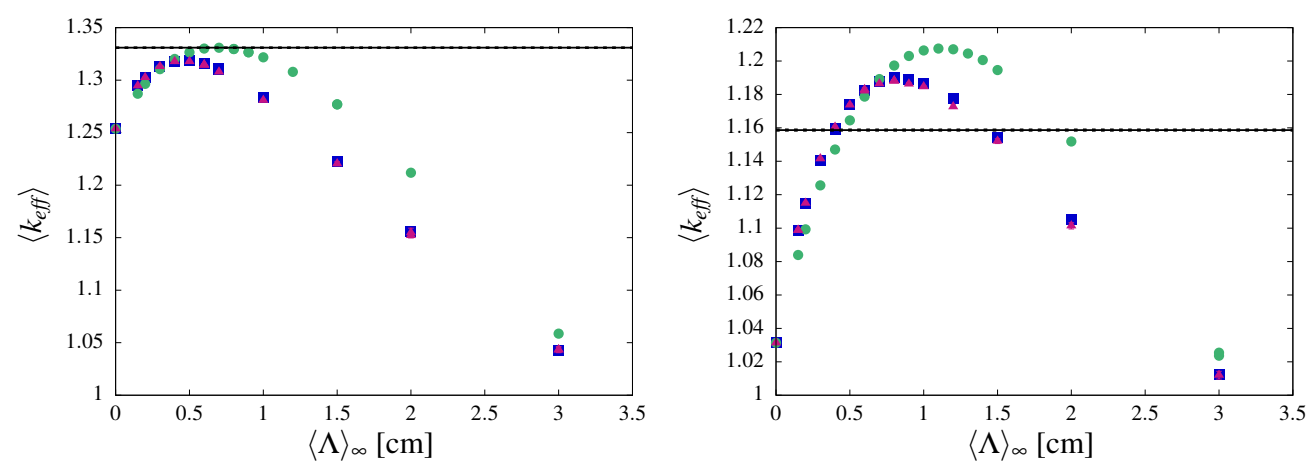

Figure 8: Evolution of the ensemble-averaged multiplication factor $\left\langle k_{\text {eff }}\right\rangle$ as a function of the average chord length $\langle\Lambda\rangle_{\infty}$. Left: UOX assembly with $n=17$. Right: MOX assembly with $n=17$. Blue squares denote the results for Poisson tessellations, red triangles for Box tessellations, and green circles for Voronoi tessellations. The limit case at $\langle\Lambda\rangle_{\infty}=0$ corresponds to the atomic mix model. The black solid line denotes $k_{\text {eff, },}$, the result for the assembly with intact fuel pins, which has been added for reference (dashed lines represent the $1 \sigma$ statistical uncertainty).

$500 \mathrm{pcm}$.

The behaviour of the case $n=17$, where the entire collection of fuel pins in the assembly is fragmented, is similar to that of the case $n=7$. The position of the maxima of $\left\langle k_{e f f}\right\rangle$ as a function of the average chord length $\langle\Lambda\rangle_{\infty}$ is almost unchanged. The range of excursion of $\left\langle k_{e f f}\right\rangle$ in the explored domain is nonetheless much larger. The eigenvalue corresponding to the atomic mix limit is lower by about $5000 \mathrm{pcm}$ for the UOX case, and by about $12000 \mathrm{pcm}$ for the MOX case. For UOX, the maxima of $\left\langle k_{\text {eff }}\right\rangle$ fall below (for Poisson and Box tessellations) or slightly above (for Voronoi tessellations) the reference $k_{\text {eff, },}$. For MOX, the maxima exceed $k_{e f f, 0}$ by about 2000 pcm for Poisson and Box tessellations, and by about $4000 \mathrm{pcm}$ for Voronoi tessellations.

It is interesting to remark that the behaviour of $\left\langle k_{e f f}\right\rangle$ as a function of $\langle\Lambda\rangle_{\infty}$ has been examined in (Pomraning, 1999) for mono-energetic transport in a rod geometry with Poisson mixing statistics: by resorting to an ingenuous perturbative approach, it was concluded that $\left\langle k_{\text {eff }}\right\rangle \geq k_{\text {eff,am }}$ for $\langle\Lambda\rangle_{\infty} \rightarrow 0$, where $k_{\text {eff, am }}$ is the eigenvalue corresponding to a (non-stochastic) atomic mix fragmentation. This result seems to hold also in the configurations examined here. 

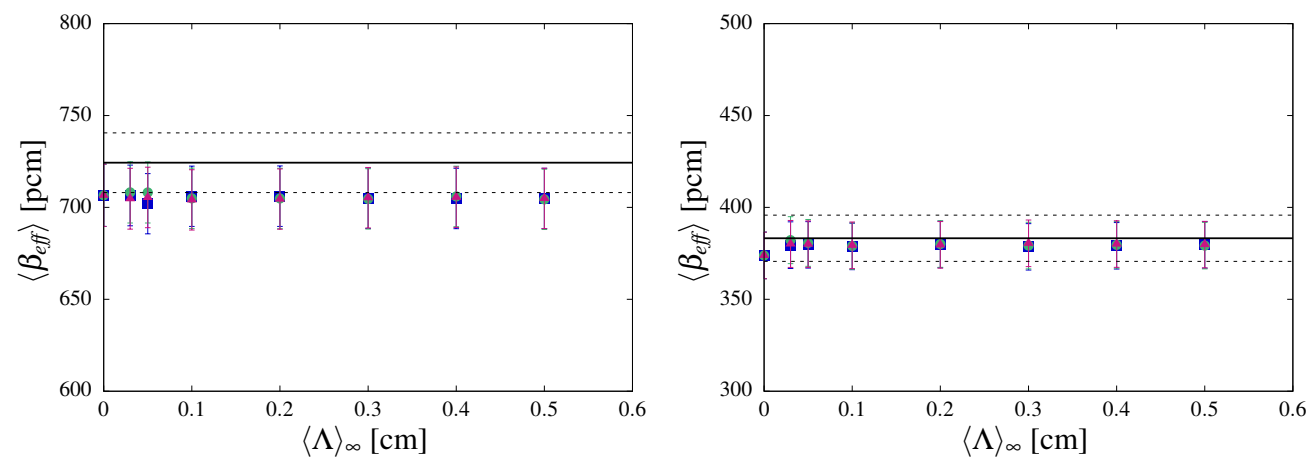

Figure 9: Evolution of the ensemble-averaged effective delayed neutron fraction $\left\langle\beta_{\text {eff }}\right\rangle$ as a function of the average chord length $\langle\Lambda\rangle_{\infty}$. Left: UOX assembly with $n=1$. Right: MOX assembly with $n=1$. Blue squares denote the results for Poisson tessellations, red triangles for Box tessellations, and green circles for Voronoi tessellations. The limit case at $\langle\Lambda\rangle_{\infty}=0$ corresponds to the atomic mix model. The black solid line denotes $\beta_{\text {eff, },}$, the result for the assembly with intact fuel pins, which has been added for reference (dashed lines represent the $1 \sigma$ statistical uncertainty).

\subsection{Delayed neutron fraction}

The evolution of the effective delayed neutron fraction $\left\langle\beta_{\text {eff }}\right\rangle$ is illustrated in Figs. 9, 10, and 11 for UOX and MOX assemblies with $n=1, n=7$ and $n=17$ melted fuel pins, respectively. The computed value $\left\langle\beta_{e f f}\right\rangle$ is displayed as a function of increasing chord length $\langle\Lambda\rangle_{\infty}$, for Poisson, Voronoi and Box tessellations. The error bar on $\left\langle\beta_{\text {eff }}\right\rangle$ is of the order of about $1 \%$ of the average, which is comparable with the uncertainty stemming from the IFP calculation for the reference assembly. The limit case of atomic mix $\left(\langle\Lambda\rangle_{\infty} \rightarrow 0\right)$ is also shown. In each figure, the $\beta_{\text {eff, },}$ eigenvalue corresponding to an assembly with intact fuel pins is plotted for reference.

For all the assembly configurations, the impact of stochastic tessellations on $\left\langle\beta_{\text {eff }}\right\rangle$ is only marginal, and in most cases well within error bars. For UOX assemblies we remark nonetheless that the random fragmentation introduces a slight bias on the average value, i.e., $\left\langle\beta_{\text {eff }}\right\rangle \leq \beta_{\text {eff, },}$, where $\beta_{\text {eff, },}$ is the reference value corresponding to an assembly with intact fuel pins. On the contrary, for

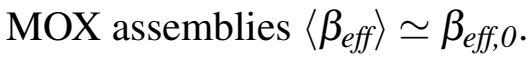

The behaviour of $\left\langle\beta_{\text {eff }}\right\rangle$ is almost unaffected by the choice of the mixing statistics. Similarly, the average chord length $\langle\Lambda\rangle_{\infty}$ plays no role, and the re- 

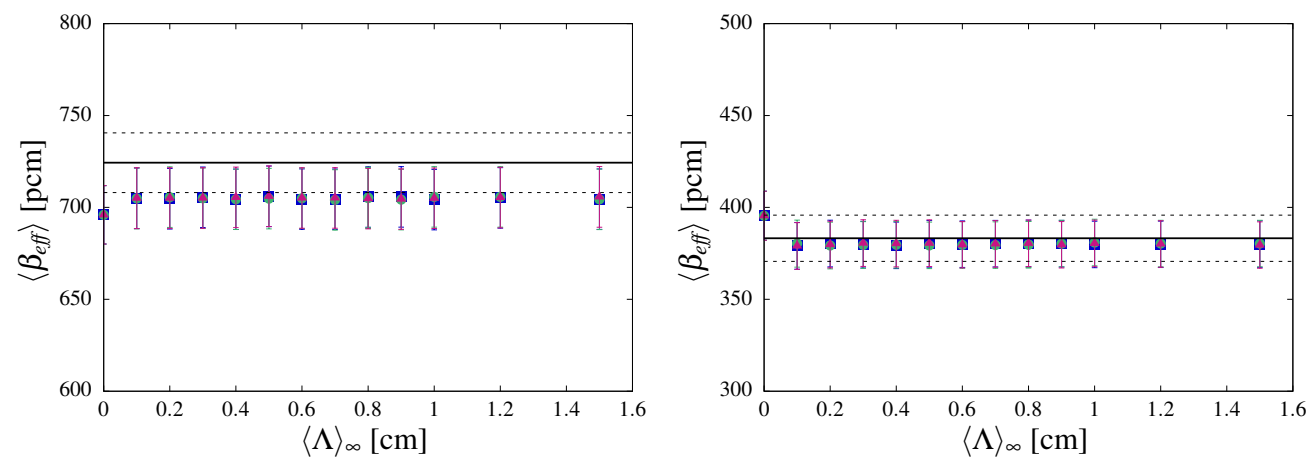

Figure 10: Evolution of the ensemble-averaged effective delayed neutron fraction $\left\langle\beta_{\text {eff }}\right\rangle$ as a function of the average chord length $\langle\Lambda\rangle_{\infty}$. Left: UOX assembly with $n=7$. Right: MOX assembly with $n=7$. Blue squares denote the results for Poisson tessellations, red triangles for Box tessellations, and green circles for Voronoi tessellations. The limit case at $\langle\Lambda\rangle_{\infty}=0$ corresponds to the atomic mix model. The black solid line denotes $\beta_{\text {eff, },}$, the result for the assembly with intact fuel pins, which has been added for reference (dashed lines represent the $1 \sigma$ statistical uncertainty).

sulting $\left\langle\beta_{\text {eff }}\right\rangle$ show a slight increasing trend only for the case $n=17$. Actually, the effective delayed neutron fraction $\left\langle\beta_{\text {eff }}\right\rangle$ depends mostly on the volumic fraction of fuel within the assembly, and this quantity is basically flat as a function of $\langle\Lambda\rangle_{\infty}$, as discussed in Sec. 4.8.

\subsection{Neutron generation time}

The evolution of the effective neutron generation time $\left\langle\ell_{\text {eff }}\right\rangle$ is illustrated in Figs. 12, 13, and 14 for UOX and MOX assemblies with $n=1, n=7$ and $n=17$ melted fuel pins, respectively. The computed value $\left\langle\ell_{\text {eff }}\right\rangle$ is displayed as a function of increasing chord length $\langle\Lambda\rangle_{\infty}$, for Poisson, Voronoi and Box tessellations. The error bar on $\left\langle\ell_{\text {eff }}\right\rangle$ is of the order of about $0.1 \%$ of the average, which is comparable with the uncertainty stemming from the IFP calculation for the reference assembly. The limit case of atomic mix $\left(\langle\Lambda\rangle_{\infty} \rightarrow 0\right)$ is also shown. In each figure, the $\ell_{\text {eff, }, 0}$ eigenvalue corresponding to an assembly with intact fuel pins is plotted for reference.

As expected, in the case $n=1$ the impact of the stochastic tessellations is small, and the discrepancy between $\left\langle\ell_{e f f}\right\rangle$ and $\ell_{e f f, O}$ lies within the error bar. For UOX assemblies, the random fragmentation induces $\left\langle\ell_{\text {eff }}\right\rangle \leq \ell_{\text {eff, },}$ for any $\langle\Lambda\rangle_{\infty}$, 

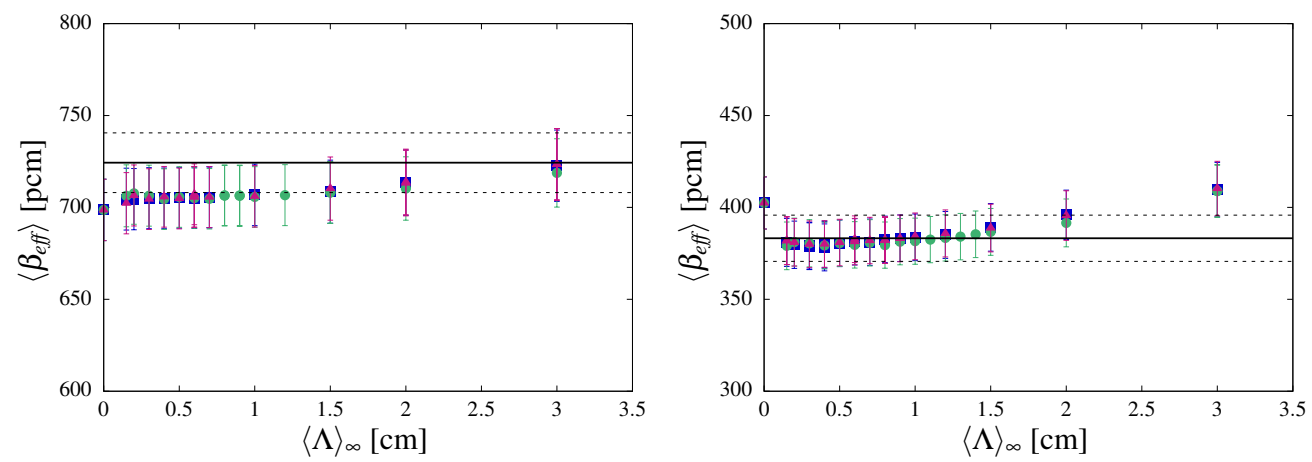

Figure 11: Evolution of the ensemble-averaged effective delayed neutron fraction $\left\langle\beta_{\text {eff }}\right\rangle$ as a function of the average chord length $\langle\Lambda\rangle_{\infty}$. Left: UOX assembly with $n=17$. Right: MOX assembly with $n=17$. Blue squares denote the results for Poisson tessellations, red triangles for Box tessellations, and green circles for Voronoi tessellations. The limit case at $\langle\Lambda\rangle_{\infty}=0$ corresponds to the atomic mix model. The black solid line denotes $\beta_{\text {eff, },}$, the result for the UOX assembly with intact fuel pins, which has been added for reference (dashed lines represent the $1 \sigma$ statistical uncertainty).

where $\ell_{\text {eff }, 0}$ is the reference value corresponding to an assembly with intact fuel pins. On the contrary, for MOX assemblies $\left\langle\ell_{\text {eff }}\right\rangle \geq \ell_{\text {eff, },}$ for any $\langle\Lambda\rangle_{\infty}$.

For the assembly configurations with $n=7$, the effects of the fuel fragmentation are clearly apparent for $\left\langle\ell_{e f f}\right\rangle$. In the atomic mix limit for small $\langle\Lambda\rangle_{\infty}$, $\left\langle\ell_{\text {eff }}\right\rangle$ lies below $\ell_{\text {eff, }, 0}$, and it gradually increases as a function of $\langle\Lambda\rangle_{\infty}$. The ensemble-averaged $\left\langle\ell_{\text {eff }}\right\rangle$ becomes larger than $\ell_{\text {eff }, 0}$ at $\langle\Lambda\rangle_{\infty} \simeq 0.3 \mathrm{~cm}$ for all mixing statistics. Poisson and Box tessellations yield almost identical results, and the corresponding $\left\langle\ell_{\text {eff }}\right\rangle$ are systematically higher than those from Voronoi tessellations. For UOX assemblies, $\left\langle\ell_{e f f}\right\rangle$ increases by about $10 \%$ in the range of $\langle\Lambda\rangle_{\infty}$ explored here, whereas for MOX assemblies the increase is of the order of $20 \%$ for the same range of $\langle\Lambda\rangle_{\infty}$.

For the case $n=17$ the behaviour of $\left\langle\ell_{\text {eff }}\right\rangle$ is qualitatively similar to that of $n=7$, but the excursion range as a function of $\langle\Lambda\rangle_{\infty}$ is wider. In particular, for UOX assemblies $\left\langle\ell_{\text {eff }}\right\rangle$ increases by about $300 \%$ in the range of $\langle\Lambda\rangle_{\infty}$ explored here, whereas for MOX assemblies the increase is of the order of $600 \%$ for the same range of $\langle\Lambda\rangle_{\infty}$. As in the previous case, Poisson and Box tessellations yield results for $\left\langle\ell_{e f f}\right\rangle$ that lie systematically higher than those of Voronoi tessellations. 

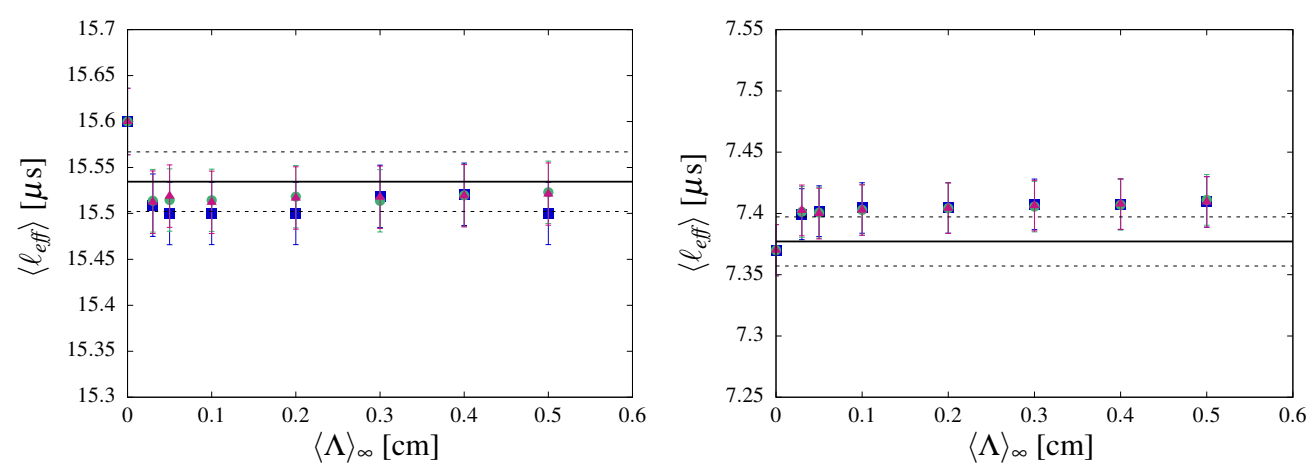

Figure 12: Evolution of the ensemble-averaged effective neutron generation time $\left\langle\ell_{\text {eff }}\right\rangle$ as a function of the average chord length $\langle\Lambda\rangle_{\infty}$. Left: UOX assembly with $n=1$. Right: MOX assembly with $n=1$. Blue squares denote the results for Poisson tessellations, red triangles for Box tessellations, and green circles for Voronoi tessellations. The limit case at $\langle\Lambda\rangle_{\infty}=0$ corresponds to the atomic mix model. The black solid line denotes $\ell_{\text {eff, },}$, the result for the UOX assembly with intact fuel pins, which has been added for reference (dashed lines represent the $1 \sigma$ statistical uncertainty).

\subsection{Distribution of the multiplication factor}

In the previous sections we have focused on the ensemble-averaged physical observables $\left\langle k_{\text {eff }}\right\rangle,\left\langle\beta_{\text {eff }}\right\rangle$, and $\left\langle\ell_{\text {eff }}\right\rangle$, and their evolution as a function of the mean chord length for different mixing statistics. In order to fully apprehend the dispersion of the multiplication factors around their average values due to the variability of the random geometry realizations, which is key for criticality safety applications, we have also computed the histograms $\Pi\left(k_{\text {eff }}\right)$. Some representative distributions are displayed in Fig. 15 as a function of $\langle\Lambda\rangle_{\infty}$ for a Poisson tessellation and in Fig. 16 as a function of the mixing statistics for fixed $\langle\Lambda\rangle_{\infty}$.

Figure 15 shows that the shape of the $\Pi\left(k_{\text {eff }}\right)$ distribution is sensitive to the average chord length: when $\langle\Lambda\rangle_{\infty}$ is small, $\Pi\left(k_{\text {eff }}\right)$ is almost Gaussian, with a small dispersion around the average $\left\langle k_{\text {eff }}\right\rangle$; as $\langle\Lambda\rangle_{\infty}$ increases, the dispersion increases, and $\Pi\left(k_{\text {eff }}\right)$ becomes less symmetric (in particular, a long left tail appears for large values of $\langle\Lambda\rangle_{\infty}$ ).

Figure 16 shows the impact of the mixing statistics on the shape of $\Pi\left(k_{e f f}\right)$, for a given average chord length $\langle\Lambda\rangle_{\infty}$. It is apparent that the stochastic tessellations affect not only the average values $\left\langle k_{e f f}\right\rangle$, but also their dispersion. In particular, for the example considered here, The Voronoi tessellation leads to a 

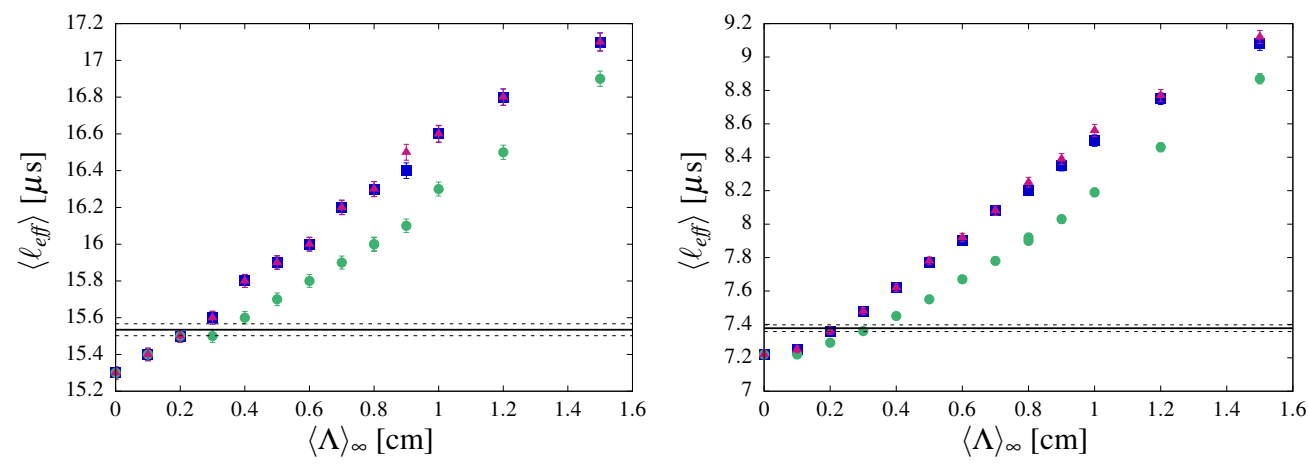

Figure 13: Evolution of the ensemble-averaged effective neutron generation time $\left\langle\ell_{\text {eff }}\right\rangle$ as a function of the average chord length $\langle\Lambda\rangle_{\infty}$. Left: UOX assembly with $n=7$. Right: MOX assembly with $n=7$. Blue squares denote the results for Poisson tessellations, red triangles for Box tessellations, and green circles for Voronoi tessellations. The limit case at $\langle\Lambda\rangle_{\infty}=0$ corresponds to the atomic mix model. The black solid line denotes $\ell_{\text {eff, },}$, the result for the UOX assembly with intact fuel pins, which has been added for reference (dashed lines represent the $1 \sigma$ statistical uncertainty).

Gaussian distribution rather peaked around the average value, whereas the Poisson and Box tessellations (whose $\Pi\left(k_{e f f}\right)$ are almost identical) lead to more dispersed and asymmetric distributions, with a long left tail.

\subsection{Scalar neutron flux}

We finalize our analysis by considering the effects of fuel fragmentation on the ensemble-averaged and normalized scalar neutron flux $\langle\varphi(\mathbf{r}, E)\rangle$. For our Monte Carlo simulations, we have defined a $17 \times 17 x-y$ spatial mesh superposed to the fuel pin-cells, with a single mesh along the $z$ axis. For symmetry reasons, the flux in the reference assemblies should be spatially flat, due to reflective boundary conditions. As for the energy dependence, we have considered a 281 group mesh, covering the entire energy range of the simulation, namely $10^{-5} \mathrm{eV}$ to $20 \mathrm{MeV}$.

535 The spatial behaviour of the neutron flux $\langle\varphi(\mathbf{r})\rangle$ is shown in Fig. 17 for $n=1$ in some representative UOX and MOX assemblies, respectively, and in Fig. 18 for $n=7$ in some representative UOX and MOX assemblies. These curves have been obtained by integrating $\langle\varphi(\mathbf{r}, E)\rangle$ over the entire energy range. The case $n=17$ leads to a spatially flat neutron flux (the fragmentation is homogeneous 

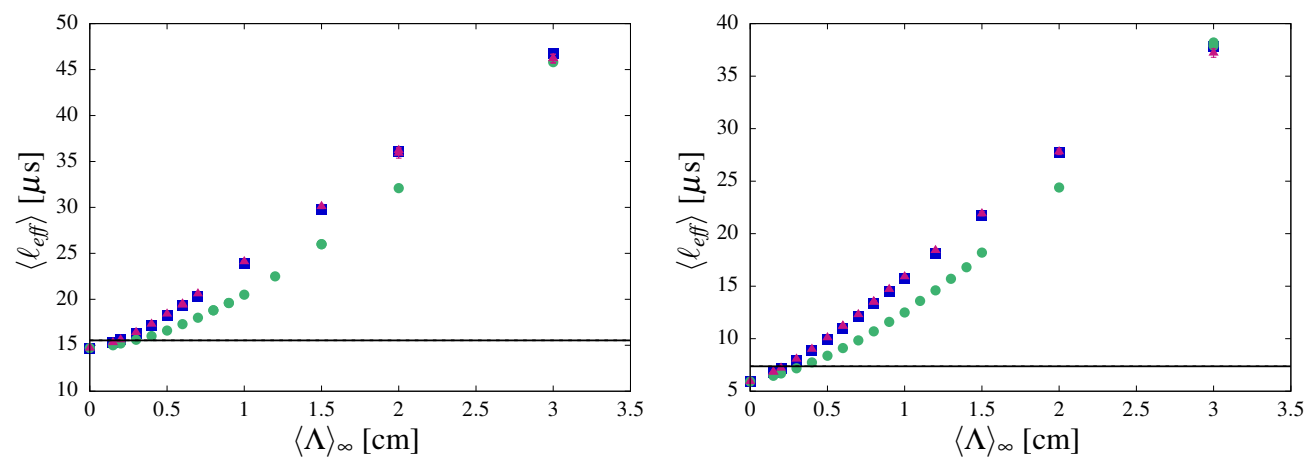

Figure 14: Evolution of the ensemble-averaged effective neutron generation time $\left\langle\ell_{\text {eff }}\right\rangle$ as a function of the average chord length $\langle\Lambda\rangle_{\infty}$. Left: UOX assembly with $n=17$. Right: MOX assembly with $n=17$. Blue squares denote the results for Poisson tessellations, red triangles for Box tessellations, and green circles for Voronoi tessellations. The limit case at $\langle\Lambda\rangle_{\infty}=0$ corresponds to the atomic mix model. The black solid line denotes $\ell_{\text {eff, },}$, the result for the UOX assembly with intact fuel pins, which has been added for reference (dashed lines represent the $1 \sigma$ statistical uncertainty).

and extended to the whole assembly) and will not be shown here. For all the examples discussed here we have considered Poisson stochastic tessellations.

For $n=1$, the effects of the stochastic tessellations on the spatial shape of the neutron flux are small, and mostly extended to a neighbourhood of the fragmented fuel pin-cell (see Fig. 17). The impact is slightly larger for MOX than for UOX assemblies. The sign of the perturbation with respect to the remaining portion of the assembly evolves as a function of $\langle\Lambda\rangle_{\infty}$ : for small $\langle\Lambda\rangle_{\infty}$ the ensemble-averaged flux close to the fragmented fuel cell lies below the value for the rest of the assembly, whereas for larger $\langle\Lambda\rangle_{\infty}$ the flux close to the fragmented fuel cell lies above. The value of $\langle\Lambda\rangle_{\infty}$ for which the ensemble-averaged spatial flux is entirely flat (i.e., the stochastic tessellation has no visible effect on the flux) corresponds approximately to the average chord length through a fuel pin. In other words, if the fragmentation of the random geometry is such that neutron trajectories see a homogeneous region whose average behaviour is statistically compatible with the heterogeneous regions of the intact fuel cell, then the neutron flux becomes insensitive to the fragmentation.

When $n=7$ (Fig. 18), the behaviour of the spatial flux is qualitatively similar to the previous case. The amplitude of the perturbations introduced by the 


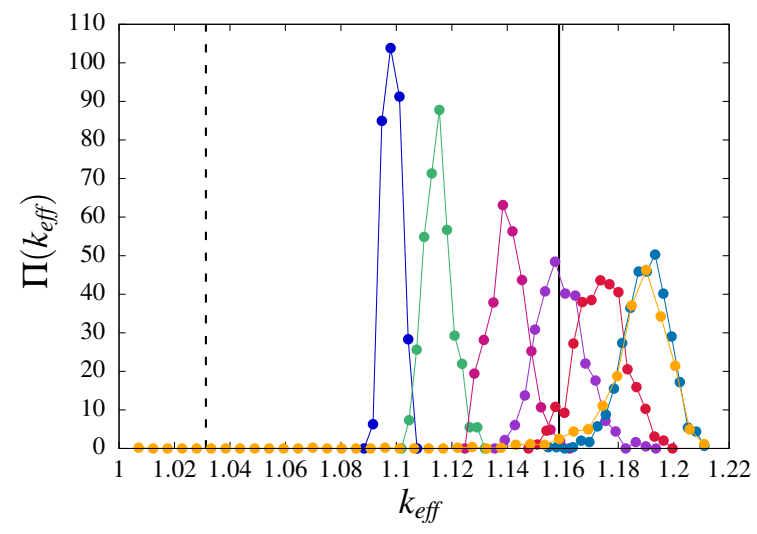

Figure 15: Distributions of the multiplication factor $k_{\text {eff }}$ as a function of the average chord length $\langle\Lambda\rangle_{\infty}$ for a MOX assembly with $n=17$. The mixing statistics is a Poisson tessellation. Blue symbols correspond to $\langle\Lambda\rangle_{\infty}=0.15$, green symbols to $\langle\Lambda\rangle_{\infty}=0.2$, magenta symbols to $\langle\Lambda\rangle_{\infty}=0.3$, purple symbols to $\langle\Lambda\rangle_{\infty}=0.4$, red symbols to $\langle\Lambda\rangle_{\infty}=0.5$, light blue symbols to $\langle\Lambda\rangle_{\infty}=0.8$ and orange symbols to $\langle\Lambda\rangle_{\infty}=1$. Solid black line corresponds to $k_{\text {eff, },}$ for a MOX assembly with intact fuel pins; dashed black line corresponds to $k_{\text {eff }}$ for a MOX assembly with atomic mix.

stochastic tessellations is larger, and the effect is extended on a larger portion of the assembly. Similarly as for $n=1$, the MOX assemblies are more sensitive to the perturbation. Again, the sign of the perturbation with respect to the remaining portion of the assembly depends evolves as a function of $\langle\Lambda\rangle_{\infty}$ : for small $\langle\Lambda\rangle_{\infty}$ the ensemble-averaged flux close to the fragmented portion of the assembly lies below the value for the rest of the assembly, whereas for larger $\langle\Lambda\rangle_{\infty}$ the perturbed flux lies above. As before, there exists a value of $\langle\Lambda\rangle_{\infty}$ for which the ensemble-averaged spatial flux is entirely flat.

Concerning the behaviour of the neutron flux with respect to energy, in Fig. 19 we show the spatially-integrated and normalized $\langle\varphi(E)\rangle$ for UOX and MOX assemblies. We have chosen the case $n=17$ with a Poisson stochastic tessellation. The impact of stochastic tessellations on $\langle\varphi(E)\rangle$ is particularly apparent when examining the discrepancies with respect to the reference flux that is obtained for the assemblies with intact fuel pins (see Fig. 20), for both UOX and MOX assemblies. The effects on $\langle\varphi(E)\rangle$ vary as a function of $\langle\Lambda\rangle_{\infty}$. For small $\langle\Lambda\rangle_{\infty}$, $\langle\varphi(E)\rangle$ lies below the reference flux in the thermal region and above for the epithermal and fast regions. For larger $\langle\Lambda\rangle_{\infty},\langle\varphi(E)\rangle$ lies above the reference flux 


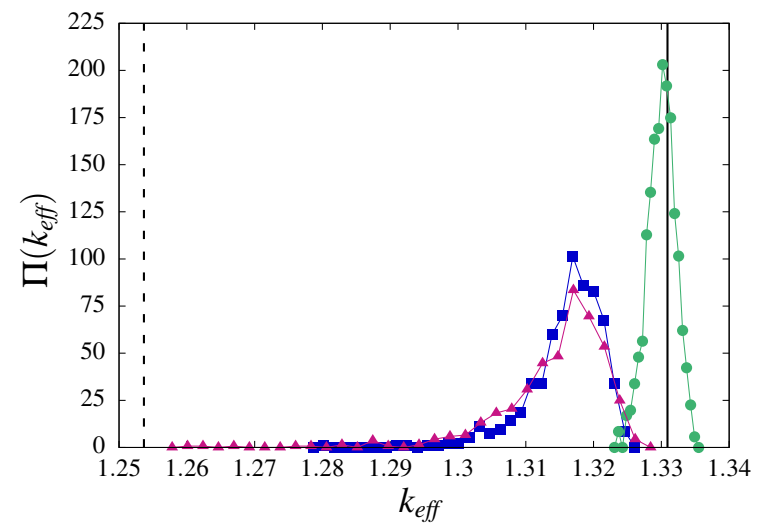

Figure 16: Distributions of the multiplication factor $k_{\text {eff }}$ for a UOX assembly with $n=17$ and different mixing statistics. The average chord length is $\langle\Lambda\rangle_{\infty}=0.6$. Blue symbols correspond to a Poisson stochastic tessellation, green symbols to Voronoi stochastic tessellations and red symbols to Box stochastic tessellations. Solid black line corresponds to $k_{\text {eff, },}$ for a UOX assembly with intact fuel pins; dashed black line corresponds to $k_{\text {eff }}$ for a UOX assembly with atomic mix.

575 in the thermal region and below for the epi-thermal and fast regions.

\subsection{Finite-size effects for the assembly calculations}

An investigation of finite-size effects for the stochastic tessellations used above has been carried out for $\left\langle\Lambda_{i}\right\rangle$, the average chord length through clusters with material composition $i$. For illustration, in Fig. 21 we show the case of 580 the assembly configurations with $n=17$, where $\left\langle\Lambda_{i}\right\rangle$ is plotted as a function of $\langle\Lambda\rangle_{\infty}$ for Poisson tessellations. As $\langle\Lambda\rangle_{\infty}$ increases, the value of $\left\langle\Lambda_{i}\right\rangle$ obtained by Monte Carlo simulation progressively deviates from the theoretical behaviour $\left\langle\Lambda_{i}\right\rangle_{\infty}=\langle\Lambda\rangle_{\infty} /\left(1-p_{i}\right)$.

We have also computed the average volumic fraction $\left\langle p_{i}\right\rangle$ through clusters 585 of composition $i$, as a function of the average chord length $\langle\Lambda\rangle_{\infty}$. The comparison with the theoretical behaviour $p_{i}$ (which is strictly valid only for infinite tessellations) is shown in Fig. 22 for an assembly configuration with $n=17$ : the deviation with respect to the ideal case increases with increasing $\langle\Lambda\rangle_{\infty}$, as expected. In order to emphasize the role of finite-size effects, in Fig. 22 we have chosen to show the geometry-induced standard deviation $\sigma_{G}$ on $p_{i}$, as given in Eq. (6), instead of the uncertainty given by Eq. 8 . 

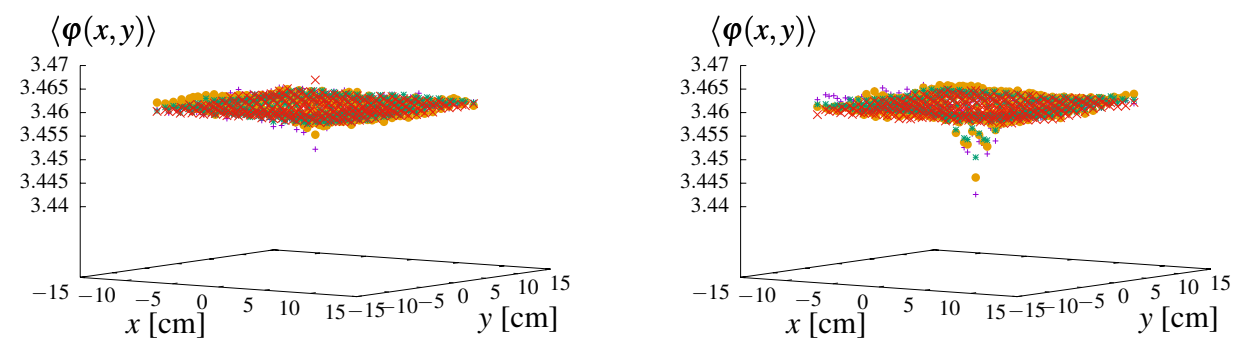

Figure 17: Ensemble-averaged spatial flux $\langle\varphi(x, y)\rangle$ (arbitrary units) as a function of the average chord length $\langle\Lambda\rangle_{\infty}$, with Poisson mixing statistics. Left: UOX assembly with $n=1$. Purple symbols correspond to $\langle\Lambda\rangle_{\infty}=0.03$, orange symbols to $\langle\Lambda\rangle_{\infty}=0.05$, green symbols to $\langle\Lambda\rangle_{\infty}=0.1$ and red symbols to $\langle\Lambda\rangle_{\infty}=0.5$. Right: MOX assembly with $n=1$. Purple symbols correspond to $\langle\Lambda\rangle_{\infty}=0.03$, orange symbols to $\langle\Lambda\rangle_{\infty}=0.05$, green symbols to $\langle\Lambda\rangle_{\infty}=0.1$ and red symbols to $\langle\Lambda\rangle_{\infty}=0.5$.

\section{Conclusions}

Stochastic tessellations provide a convenient tool for the analysis of randomly fragmented materials. In this paper we have proposed a methodology for the analysis of the impact of random geometries in three-dimensional fuel assembly, with application to criticality safety for severe accidents. Based on a random geometry generator that we have recently developed for Poisson, Voronoi and Box tessellations, we were able to create large ensembles of UOX and MOX assembly configurations with varying portions of fragmented fuel cells. These configurations can be read by the Monte Carlo transport code TRIPOLI-4 ${ }^{\circledR}$, which has been used to compute the multiplication factor, the adjoint-weighted kinetics parameters, and the scalar neutron flux. Analysis of the resulting ensembleaveraged physical quantities has allowed assessing the impact of stochastic tessellations on the key reactor core parameters. In particular, we have determined the evolution of $\left\langle k_{e f f}\right\rangle,\left\langle\beta_{e f f}\right\rangle$, and $\left\langle\ell_{\text {eff }}\right\rangle$ as a function of the mean chord length of the random geometries, which is related to the correlation length of the fragmented portion of the assembly. The effect of varying the mixing statistics has been also examined: while Poisson and Box tessellations yield almost identical results, Voronoi tessellations yield distinct results. These findings show that the three mixing statistics, while sharing the same average chord length by con- 

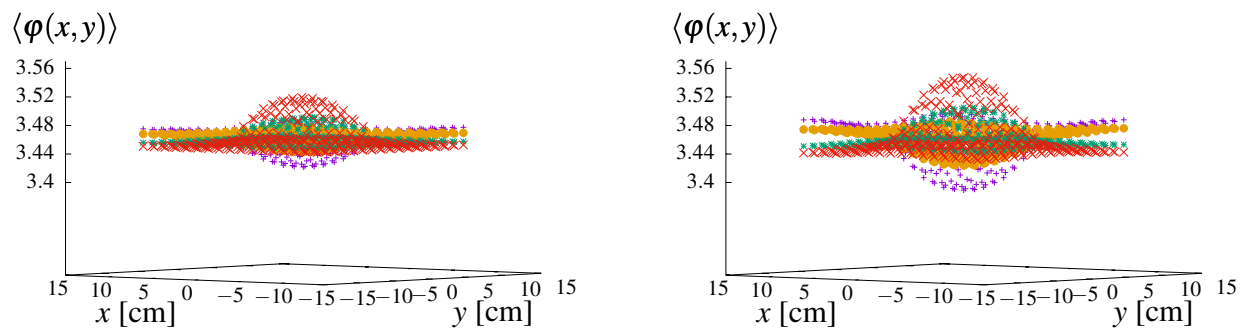

Figure 18: Ensemble-averaged spatial flux $\langle\varphi(x, y)\rangle$ (arbitrary units) as a function of the average chord length $\langle\Lambda\rangle_{\infty}$, with Poisson mixing statistics. Left: UOX assembly with $n=7$. Purple symbols correspond to $\langle\Lambda\rangle_{\infty}=0.1$, orange symbols to $\langle\Lambda\rangle_{\infty}=0.2$, green symbols to $\langle\Lambda\rangle_{\infty}=0.5$ and red symbols to $\langle\Lambda\rangle_{\infty}=0.7$. Right: MOX assembly with $n=7$. Purple symbols correspond to $\langle\Lambda\rangle_{\infty}=0.1$, orange symbols to $\langle\Lambda\rangle_{\infty}=0.2$, green symbols to $\langle\Lambda\rangle_{\infty}=0.5$ and red symbols to $\langle\Lambda\rangle_{\infty}=0.7$.

truction, might yet induce subtle effects on neutron transport due tho the precise shape of their associated chord length distributions. Generally speaking, MOX assemblies seem more sensitive than UOX assemblies to the perturbations introduced by the stochastic tessellations.

The preliminary results presented in this work are admittedly too simplified to be amenable to general conclusions concerning the behaviour of a reactor core in the presence of partially melted fuel pins. In particular, we did not address the coupling between neutron transport and thermal-hydraulics (we have assumed the temperature to be constant at $300 \mathrm{~K}$ in the assembly, for conservatism), and we focused exclusively on the stationary behaviour. The complex physical-chemistry of the reactions occurring between the fuel and the cladding at high temperature have not been addressed, either. Nonetheless, our approach is fairly broad and might be extended to more complex situations. For instance, the same procedure could be applied also to the assessment of re-criticality probability of out-of-pile core degradation leading to the deposition of corium debris. Monte Carlo simulation, which is capable of dealing with arbitrarily shaped geometries, would be particularly useful in this context. 

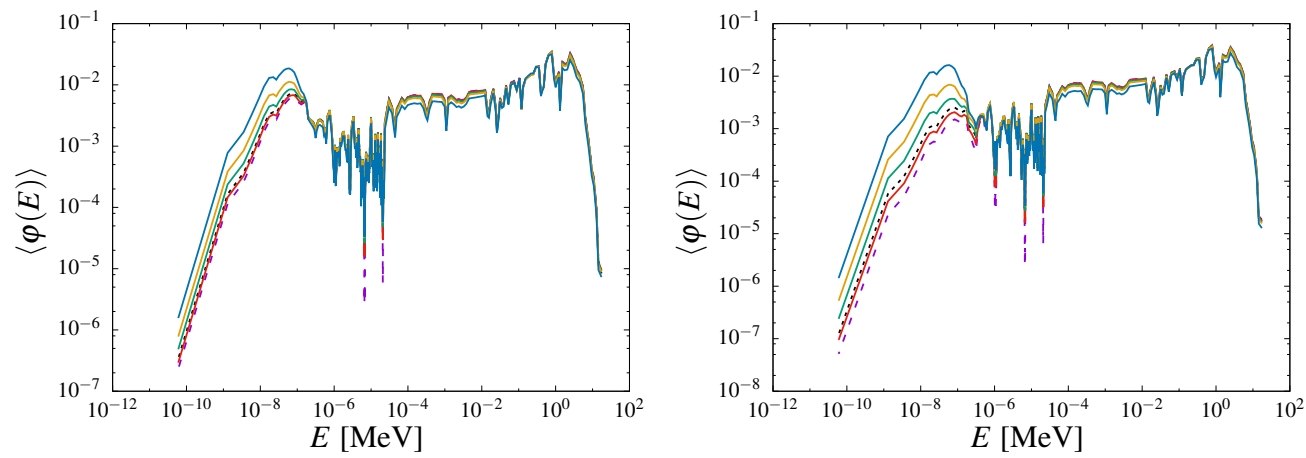

Figure 19: Ensemble-averaged spectral flux $\langle\varphi(E)\rangle$ (arbitrary units) as a function of the average chord length $\langle\Lambda\rangle_{\infty}$, with Poisson mixing statistics. Left: UOX assembly with $n=17$. Red solid line corresponds to $\langle\Lambda\rangle_{\infty}=0.15$, green solid line to $\langle\Lambda\rangle_{\infty}=0.5$, orange solid line to $\langle\Lambda\rangle_{\infty}=1$ and blue solid line to $\langle\Lambda\rangle_{\infty}=3$. Right: MOX assembly with $n=17$. Red solid line corresponds to $\langle\Lambda\rangle_{\infty}=0.15$, green solid line to $\langle\Lambda\rangle_{\infty}=0.5$, orange solid line to $\langle\Lambda\rangle_{\infty}=1$ and blue solid line to $\langle\Lambda\rangle_{\infty}=3$. For both UOX and MOX configurations, the dotted black line corresponds to $\langle\varphi(E)\rangle$ for assemblies with intact fuel pins; the dashed purple line corresponds to $\langle\varphi(E)\rangle$ for assemblies with atomic mix.

\section{Acknowledgements}

TRIPOLI- $4{ }^{\circledR}$ is a registered trademark of CEA. The authors wish to thank Électricité de France (EDF) for partial financial support.

Adams, M.L., Larsen, E.W., Pomraning, G.C., 1989. J. Quant. Spectrosc. Radiat. Transfer 42, 253-266.

Ambos, A.Yu., Mikhailov, G.A., 2011. Russ. J. Numer. Anal. Math. Modelling 26, 263-273.

Brantley, P.S., Palmer, T.S., 2009. In Proceedings of the international conference on mathematics, computational methods \& reactor physics (M\&C2009), Saratoga Springs, New York.

Brantley, P.S., 2009. In Proceedings of the international conference on mathematics, computational methods \& reactor physics (M\&C2009), Saratoga Springs, New York.

Brantley, P.S., 2011. J. Quant. Spectrosc. Radiat. Transfer 112, 599-618.

Broughton, J.M., Kuan, P., Petti, D.A., Tolman, E.L., 1989. Nucl. Techn. 87, 34-53.

640 Brown, F.B., Martin, W.R., 2004. Ann. Nucl. Energy 31, 2039-2047.

Brun, E., et al., 2015. Ann. Nucl. Energy 82, 151-160.

Chiu, S.N., Stoyan, D., Kendall, W.S., Mecke, J., 2013. Stochastic geometry and its applications, Wiley, New York, USA.

Coleman, R., 1969. J. Appl. Probab. 6, 430-441.

645 Donovan, T.J., Danon, Y., 2003. Nucl. Sci. Eng. 143, 226-239. 

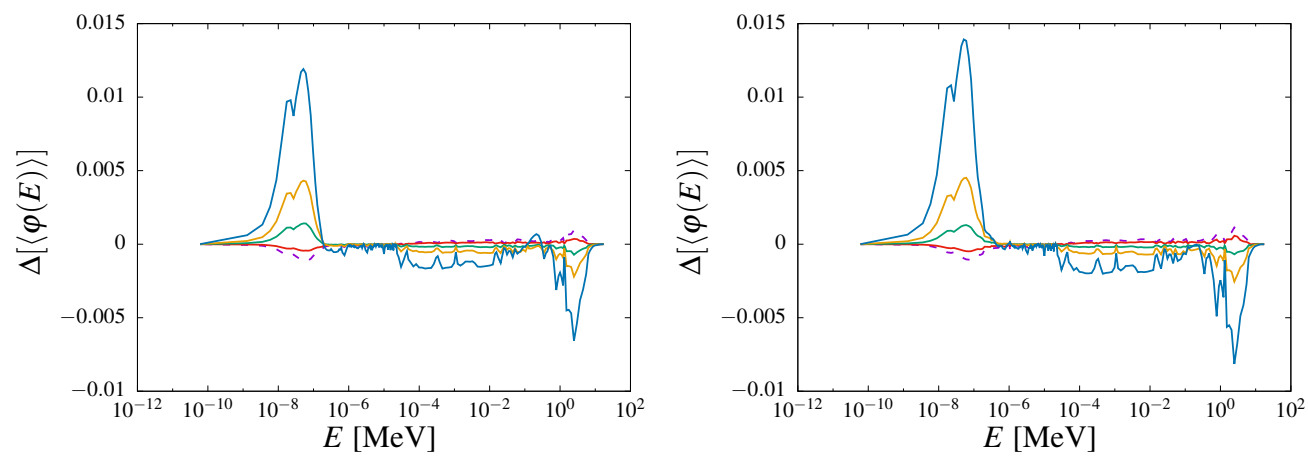

Figure 20: Discrepancy $\Delta[\langle\varphi(E)\rangle]$ between the ensemble-averaged spectral flux $\langle\varphi(E)\rangle$ (arbitrary units) as a function of the average chord length $\langle\Lambda\rangle_{\infty}$ and the reference flux, for Poisson mixing statistics. Left: UOX assembly with $n=17$. Red solid line corresponds to $\langle\Lambda\rangle_{\infty}=0.15$, green solid line to $\langle\Lambda\rangle_{\infty}=0.5$, orange solid line to $\langle\Lambda\rangle_{\infty}=1$ and blue solid line to $\langle\Lambda\rangle_{\infty}=3$. Right: MOX assembly with $n=17$. Red solid line corresponds to $\langle\Lambda\rangle_{\infty}=0.15$, green solid line to $\langle\Lambda\rangle_{\infty}=0.5$, orange solid line to $\langle\Lambda\rangle_{\infty}=1$ and blue solid line to $\langle\Lambda\rangle_{\infty}=3$. For both MOX and UOX configurations, the dotted black line corresponds to $\langle\varphi(E)\rangle$ for assemblies with intact fuel pins; the dashed purple line corresponds to $\langle\varphi(E)\rangle$ for assemblies with atomic mix.

Donovan, T.J., Sutton, T.M., Danon, Y., 2003. In Proceedings of the nuclear mathematical and computational sciences: a century in review, a century anew, Gatlinburg, TN.

Doub, W.B., 1961. Nucl. Sci. Eng. 10, 299-307.

Gilbert, E.N., 1962. Ann. Math. Statist. 33, 958-972.

650 Gomit, J.M., et al., 2011. In Proceedings of the ICNC 2011 conference, Edinburgh, Scotland.

Griesheimer, D.P., Millman, D.L., Willis, C.R., 2010. J. Quant. Spectrosc. Radiat. Transfer 112, 577598.

Hagen, S., Hofmann, P., 1987. Nucl. Eng. Design 103, 85-106.

Haran, O., Shvarts, D., Thieberger, R., 2000. Phys. Rev. E 61, 6183-6189.

655 Hofmann, P., 1999. J. Nucl. Mat. 270, 194-211.

Kiedrowski, B. C., Brown, F. B., Wilson, P. P. H., 2011b. Nucl. Sci. Eng. 68, 226.

Ji, W., Martin, W.R., 2011. Nucl. Sci. Eng. 169, 19-39.

Larmier, C., Dumonteil, E., Malvagi, F., Mazzolo, A., Zoia, A., 2016. Phys. Rev. E 94, 012130.

Larmier, C., Hugot, F.-X., Malvagi, F., Mazzolo, A., Zoia, A., 2017. J. Quant. Spectrosc. Radiat. Transfer 189, 133148.

Larmier, C., Zoia, A., Malvagi, F., Dumonteil, E., Mazzolo, A., 2017. To be appear in J. Quant. Spectrosc. Radiat. Transfer.; see https : / / arxiv.org/abs/1702.00049.

Larsen, E.W., Vasques, R., 2011. J. Quant. Spectrosc. Radiat. Transfer. 112, 619-631.

Lepage, T., Delaby, L., Malvagi, F., Mazzolo, A., 2011. Prog. Nucl. Sci. Techn. 2, 743-748. 


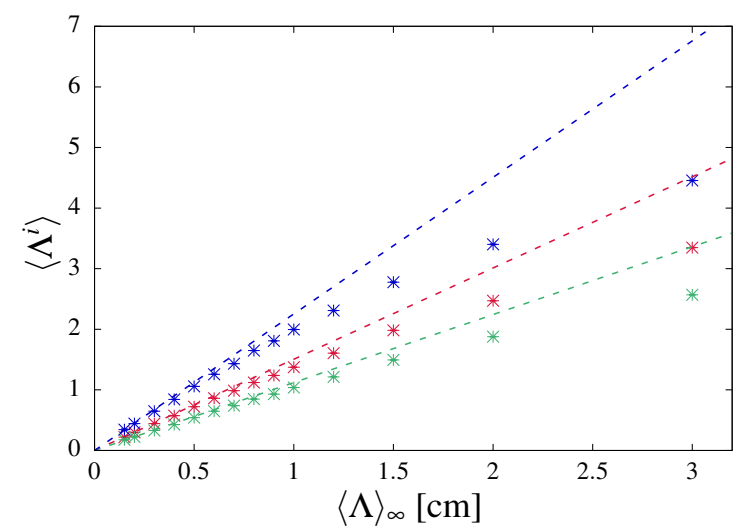

Figure 21: Average chord length $\left\langle\Lambda^{i}\right\rangle$ through clusters of composition $i$, as a function of $\langle\Lambda\rangle_{\infty}$, for an assembly with $n=17$. The case of fuel clusters is displayed in red, the case of cladding clusters in green and the case of moderator clusters in blue. Symbols correspond to Monte Carlo simulations. Theoretical values for infinite tessellations are displayed as dashed lines.

Levermore, C.D., Pomraning, G.C., Sanzo, D.L., Wong, J., 1986. J. Math. Phys. 27, 2526-2536. Liang, C., Ji, W., Brown, F.B., 2013. Ann. Nucl. Energy 53, 140-146.

Malvagi, F., Byrne, R.N., Pomraning, G.C., Somerville, R.C.J., 1992. J. Atm. Sci. 50, 2146-2158.

McLane, V., 2004. Brookhaven National Laboratory report BNL-NCS-44945-01/04-REV KB0301042.

670 Meijering, J.L., 1953. Philips Res. Rep. 8, 270-290.

Miles, R.E., 1970. Izv. Akad. Nauk. Arm. SSR Ser. Mat. 5, 263-285.

Miles, R.E., 1972. Suppl. Adv. Appl. Prob. 4, 243-266.

Murata, I., Mori, T., Nakagawa, M., 1996. Nucl. Sci. Eng. 123, 96-109.

Nauchi, Y., Kameyama, T., 2010. J. Nucl. Sci. Technol. 47, 977.

675 Pomraning, G.C., 1991. Linear kinetic theory and particle transport in stochastic mixtures, World Scientific Publishing, River Edge, NJ, USA.

Pomraning, G.C., 1999. Ann. Nucl. Energy 26, 217-235.

Santaló, L.A., 1976. Integral geometry and geometric probability, Addison-Wesley, Reading, MA, USA.

680 Santamarina, A., et al., (2009). The JEFF-3.1.1 Nuclear Data Library, JEFF Report 22, OECDNEA Data Bank.

Serra, J., 1982. Image analysis and mathematical morphology, Academic Press, London, UK. Stauffer, D., Aharony, A., 1994. Introduction To Percolation Theory, CRC Press., FL, USA.

Su, B., Pomraning, G.C., 1995. J. Quant. Spectrosc. Radiat. Transfer 54, 779-801.

685 Sublet, J.C., et al., 2011. CALENDF-2010: User Manual, Rapport CEA-R-6277.

Torquato, S., 2013. Random heterogeneous materials: microstructure and macroscopic properties, Springer-Verlag, New York, USA.

Truchet, G., Leconte, P., Santamarina, A., Damian, F., Brun, E., Zoia, A., 2015. Ann. Nuc. 


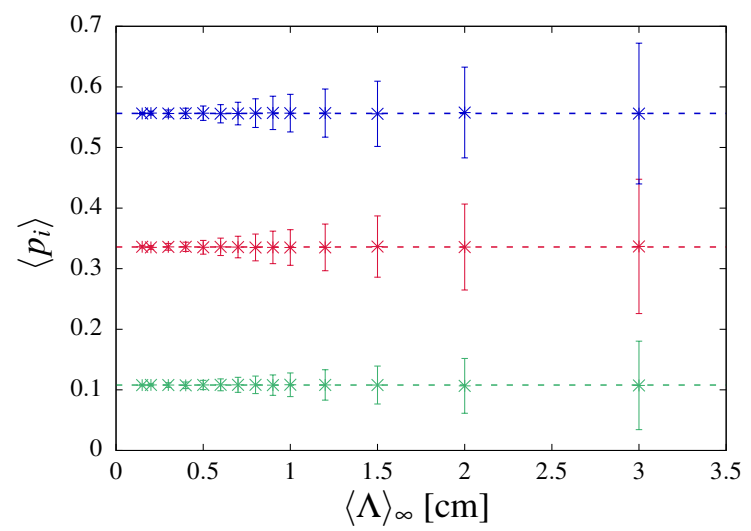

Figure 22: Average volumic fraction $\left\langle p_{i}\right\rangle$ through clusters of composition $i$, as a function of $\langle\Lambda\rangle_{\infty}$, for an assembly with $n=17$. Red symbols denote the fuel fraction, green symbols the cladding fraction and blue symbols the moderator fraction. Symbols correspond to Monte Carlo simulations. Error bars on Monte Carlo results represent the standard deviation $\sigma_{G}$ on $p_{i}$, as given in Eq. (6). Theoretical values for infinite tessellations are displayed as dashed lines.

Energy 85, 17.

690 Williams, M.M.R., 2000. Nucl. Sci. Eng. 136, 34-58.

Williams, M.M.R., Larsen, E.W., 2001. Nucl. Sci. Eng. 139, 66-77.

Williams, M.M.R., 2003. Nucl. Sci. Eng. 143, 1-18.

Yamamoto, T., 2010. Ann. Nucl. Energy 37, 398-405.

Zimmerman, G.B., 1990. Lawrence Livermore National Laboratory Report UCRL-JC-105616.

695 Zimmerman, G.B., Adams, M.L., 1991, Trans. Am. Nucl. Soc. 66, 287.

Zoia, A., Brun, E., Jouanne, C., Malvagi, F., 2012. Ann. Nucl. Energy 54, 218-226.

Zoia, A., Brun, E., Malvagi, F., 2014. Ann. Nucl. Energy 63, 276.

Zoia, A., Brun, E., Damian, F., Malvagi, F., 2015. Ann. Nucl. Energy 75, 627.

Zoia, A., Brun, E., 2016. Annals of Nuclear Energy 90, 71-82.

700 Zoia, A., Nauchi, Y., Brun, E., Jouanne, C., 2016. Annals of Nuclear Energy 96, 377-388.

Zuchuat, O., Sanchez, R., Zmijarevic, I., Malvagi, F., 1994. J. Quant. Spectrosc. Radiat. Transfer 51 689-722. 